\title{
Causality and stability conditions of a conformal charged fluid
}

\section{Farid Taghinavaz}

School of Particles and Accelerators, Institute for Research in Fundamental Sciences (IPM), P.O. Box 19395-5531, Tehran, Iran

E-mail: ftaghinavaz@ipm.ir

ABSTRACT: In this paper, I study the conditions imposed on a normal charged fluid so that the causality and stability criteria hold for this fluid. I adopt the newly developed General Frame (GF) notion in the relativistic hydrodynamics framework which states that hydrodynamic frames have to be fixed after applying the stability and causality conditions. To do this, I take a charged conformal matter in the flat and $3+1$ dimension to analyze better these conditions. The causality condition is applied by looking to the asymptotic velocity of sound hydro modes at the large wave number limit and stability conditions are imposed by looking to the imaginary parts of hydro modes as well as the Routh-Hurwitz criteria. By fixing some of the transports, the suitable spaces for other ones are derived. I observe that in a dense medium having a finite $\mathrm{U}(1)$ charge with chemical potential $\mu_{0}$, negative values for transports appear and the second law of thermodynamics has not ruled out the existence of such values. Sign of scalar transports are not limited by any constraints and just a combination of vector transports is limited by the second law of thermodynamic. Also numerically it is proved that the most favorable region for transports $\tilde{\gamma}_{1,2}$, coefficients of the dissipative terms of the current, is of negative values.

Keywords: Thermal Field Theory, Quark-Gluon Plasma

ARXIV EPRINT: 2004.01897 


\section{Contents}

1 Introduction 1

2 Preliminaries 3

3 Dense fluids 5

3.1 Shear modes 8

$\begin{array}{ll}3.2 & \text { Sound modes }\end{array}$

4 Finite density fluid $\quad 16$

$\begin{array}{lll}4.1 & \text { Sound channel } & 17\end{array}$

$\begin{array}{llr}5 & \text { Conclusion } & 20\end{array}$

A On-shell entropy and negativeness of transports 22

\section{Introduction}

Relativistic Hydrodynamics (RH) is the most powerful tool to describe in and out of equilibrium properties of hot and dense QCD matter. It explains the dynamics of quark matter in terms of some local effective Degrees of Freedom (DoF) rather than infinite microscopic DoF and utilizes some tools to describe the evolution of quark matter. Examination of the $\mathrm{RH}$ has been intensified since the previous decade which experiments confirm that observables of heavy ion collision are in good agreement with predictions of the RH [1-4].

In the last few years, some experimental and analytical challenges have changed the path of the RH studies. Here, two of these challenges are addressed and my problem lies in one of these mainstreams. Traditionally, it was believed that two conditions are mandatory for the RH. The first one is the existence of a local and stable thermal equilibrium and the second one is the validity of gradient expansion. Thus, having a large number of colliding particles is necessary to achieve these goals. Otherwise, the concept of equilibrium and slow variation does not make sense. But recent observations in RHIC and LHC have suspected us about these two axioms. They are about the collective behaviors in small system collisions in which the local thermal equilibrium state and the smooth variation of macroscopic fields stop to reach [5-8]. These observations have pushed theoretical works to study the late time behavior of QCD matter dynamics. There is a large literature in this field which states that an attractor solution appears in the hydrodynamics calculations regardless of any initial conditions, [9-20] and references therein. Having an attractor solution means that the $\mathrm{RH}$ can be applied to any high energetic collisions of particles -regardless of its size and at late times the "causal" RH equations can be used safely. 
Another stream in the RH is the stability and causality issues. Historically, it has been proved that first order RH suffers from the growing amplitudes of fluctuations in time (the stability problem) and the superluminal propagation speed of fluctuations (the causality problem) [21-24]. This pathology has been cured by introducing the second order terms in the entropy current vector. The inclusion of phenomenological second order terms into the first order dissipative calculations is known as the Muller-Israel-Stewart (MIS) framework. The MIS approach does not guarantee the stability and causality of hydro modes, per se. But rather, for the MIS theory to be a stable and causal formalism, it should satisfy certain conditions. For example $\eta$, the shear transport coefficient and $\tau$, the shear relaxation time are no longer independent parameters, but instead satisfy $\frac{\eta}{\tau_{\pi} T s} \leq \frac{1}{2}$ [25]. Also the local velocity of fluid's parcel and the dimension of space-time influence these conditions [26]. In the paper [27] it has been studied the effects of initial conditions and higher order terms on stability and causality of first order hydro.

Recently it appears a trend in the $\mathrm{RH}$ which does not need to include higher order gradient terms in the entropy current [28-30]. They focused on the notion of General Frame (GF) and the definition of new transport coefficients ahead of the gradient terms. In the natural process of the $\mathrm{RH}$, the concept of "frame" arises when dissipative terms enter into the calculations. This is because of the lack of definitions for thermodynamic fields such as temperature and chemical potential in out of equilibrium situations [4]. People usually have used this freedom to fix the frame, i.e. the Landau or Eckart frame, and then proceed to do the calculations. In the GF approach, the frame freedom is respected and we do not try to fix them before doing any calculations. We first perform the RH computations and since then decide which frame is physical or not. For instance, to study the stability and causality issue, we utilize the notion of GF to fix the hydrodynamical frames after computing the hydrodynamical (hydro) modes. This job has shrunk the space of transport coefficients and reduced them to those satisfy some special conditions.

The MIS and GF approaches have distinct features. The MIS lacks a fundamental base and it is only a phenomenological approach, but the GF framework is based upon a good deal with the frame notion which is a physical concept. Another difference is that the MIS enters some variables into the $\mathrm{RH}$ with an extra relaxation type equation, while in the GF approach there is no DoF besides the temperature, chemical potential and fluids velocity. Therefore, it seems that GF emerges from a renormalizable field theory while the MIS does not originate from a renormalizable theory.

So far the investigations in the GF approach are about the uncharged conformal fluid and try to limit the space of transports to the causal and stable regions. My motivation to do this work is to generalize the preceding works to a charged QCD plasma or equivalently having a finite $\mathrm{U}(1)$ chemical potential " $\mu_{0}$ ". I perform the calculations for two circumstances. The first is for a hyperdense fluid with $\mu_{0} \gg T_{0}$ and the second for a fluid with a finite $\mu_{0}$ and $T_{0}$. Both of these studies are done by the assumption of conformal symmetry imposed on the Equation of State (EoS) and other related quantities. The hyperdense medium of quark matter is believed to be as color-superconductor phase and it is not a strongly interacting plasma but studying the hydrodynamics of this medium is of great importance. By studying the hydro modes and some other quantities, I obtain the physical conditions on 
the transports which causes a conformal charged fluid to be a stable and causal theory. In the finite density medium case, this work is repeated for two values of $\frac{\mu_{0}}{T_{0}}$. The main achievement of this paper is that signs of scalar transports are not fixed by using the second law of thermodynamics $\left(\partial_{\mu} S^{\mu} \geq 0\right)$ and just sign of combination of vector transports are fixed by it. Thus, scalar transports as well as the vectors might have negative values and the second law does not rule out it anymore. This negativeness occurs for transports that are not invariant under the frame redefinition. However, importance of my result is that in charged fluid the changing transports could be negative numbers, while in a charged fluid [28] the changing transports have to be non-negative numbers. Also, I find that the conditions derived from stability and Routh-Hurwitz criteria respect to the causality conditions.

The organization of this paper is as follows. In section 2, I shall discuss some preliminary stuffs of the $\mathrm{RH}$, the idea of frame and the GF notion. In section 3, I will try to get some conditions on the transport parameters of a hyperdense fluid $\mu_{0} \gg T_{0}$ by doing the hydro modes and taking the conformal symmetry over the EoS and other quantities. By fixing five of them the physical regions for other transports are derived and the space of $\tilde{\gamma}_{2}$ and $\tilde{\epsilon}_{2}$ transports is constrained. In section 4 , I will repeat the works done in section 3 for finite $T_{0}$ and $\mu_{0}$ medium and two values of $\frac{\mu_{0}}{T_{0}}$. Eventually, I close the paper with a discussion about the conclusions and I address some problems which can be done along this paper.

Throughout the present paper, I take the $\hbar=c=k_{B}=1$ convention and the Minkowski metric is mostly positive which results in the relation $u^{\mu} u_{\mu}=-1$.

\section{Preliminaries}

Conserved currents are of great importance in a relativistic system. According to the Noether theorem, these currents belong to either the space-time or internal symmetries. Energy momentum tensor $T^{\mu \nu}(x)$ and other conserved currents such as $J^{\mu}(x)$ correspond to the space-time and internal symmetries, respectively. The dynamical evolution of these currents are given by the conservation laws

$$
\begin{gathered}
\nabla_{\mu} T^{\mu \nu}=0, \\
\nabla_{\mu} J^{\mu}=0,
\end{gathered}
$$

which are consequences of diff and gauge transformations [31]. To make consistent these EoM and number of local effective DoF, we utilize of some relations named as constitutive relations [4]

$$
\begin{aligned}
T^{\mu \nu}(x) & =\mathcal{E}(x) u^{\mu}(x) u^{\nu}(x)+\mathcal{P}(x) \Delta^{\mu \nu}(x)+Q^{\mu}(x) u^{\nu}(x)+Q^{\nu}(x) u^{\mu}(x)+t^{\mu \nu}(x), \\
J^{\mu}(x) & =\mathcal{N}(x) u^{\mu}(x)+\mathcal{J}^{\mu}(x) .
\end{aligned}
$$

In these relations, $(\mathcal{E}(x), \mathcal{P}(x), \mathcal{N}(x))$ represent the local energy density, pressure and a conserved number density, respectively. $u^{\mu}(x)$ stands for the local fluid velocity and $\Delta^{\mu \nu}(x)=$ $g^{\mu \nu}(x)+u^{\mu}(x) u^{\nu}(x)$ is an operator which projects a given tensor onto the space perpendicular to the $u^{\mu}(x) . Q^{\mu}(x)$ is the local heat current, $t^{\mu \nu}(x)$ is the traceless symmetric part of the energy momentum tensor and $\mathcal{J}^{\mu}(x)$ is an added term to the current density which appears 
in higher derivative corrections. All the aforementioned hydro fields have to be described in terms of local effective DoF. These DoF are chosen to be as the $\left(T(x), \mu(x), u^{\mu}(x)\right)$ and the local equilibrium values of these DoF are called as the thermo fields.

Apart from the constitutive relations, the derivative expansion assumption is a great tool in the RH. This assumption tells that hydro fields are expressed in terms of slowly varying thermo fields and their derivatives. The general forms of hydro fields are written as follows $[4]$

$$
\begin{array}{rlrl}
\mathcal{E} & =\epsilon_{0}+f_{\mathcal{E}}(\partial T, \partial \mu, \partial u)+\ldots, & \mathcal{P}=p_{\text {id }}+f_{\mathcal{P}}(\partial T, \partial \mu, \partial u)+\ldots, \\
\mathcal{N} & =n_{0}+f_{\mathcal{N}}(\partial T, \partial \mu, \partial u)+\ldots & & \\
\mathcal{Q}^{\mu} & =f_{\mathcal{Q}}(\partial T, \partial \mu, \partial u)+\ldots, & \mathcal{J}^{\mu}=f_{\mathcal{J}}(\partial T, \partial \mu, \partial u)+\ldots \\
t^{\mu \nu} & =f_{t}(\partial T, \partial \mu, \partial u)+\ldots & &
\end{array}
$$

Hereafter, I omit the $x$ dependence in these fields. In the zeroth order (ideal limit), equilibrium values of hydro fields are written in terms of the local values of thermo fields

$$
\epsilon_{0}=\mathcal{E}\left(T_{0}, \mu_{0}, u_{0}^{\mu}\right), \quad p_{\text {id }}=\mathcal{P}\left(T_{0}, \mu_{0}, u_{0}^{\mu}\right), \quad n_{0}=\mathcal{N}\left(T_{0}, \mu_{0}, u_{0}^{\mu}\right) .
$$

The functions $f(\partial T, \partial \mu, \partial u)$ in the relations (2.5)-(2.7) represent the first order corrections and the notation "..." stands for the higher order corrections. At higher order calculations of the hydrodynamics, the idea of frame plays an important role. It works as follows. Thermo fields have no unique definitions in the higher order corrections. It means that we can redefine them by adding new contributions

$$
T \rightarrow T+\delta T, \quad \mu \rightarrow \mu+\delta \mu, \quad u^{\mu} \rightarrow u^{\mu}+\delta u^{\mu}
$$

in such a way that energy momentum tensor and current density remain unchanged. Therefore, there is no any preferred values for these thermo fields [4]. Different redefinitions of thermo fields are usually called as the "hydro frames" and the freedom in choice of specific value for thermo fields is often called as the "frame freedom". These redefinitions resemble to the gauge freedom in QFT. Usually, the corrections $\left(\delta T, \delta \mu, \delta u^{\mu}\right)$ are written in terms of derivatives of thermo fields [28]

$$
\begin{aligned}
\delta T & =a_{1} \frac{u^{\mu} \partial_{\mu} T}{T}+a_{2} \partial_{\mu} u^{\mu}+a_{3} u^{\mu} \partial_{\mu}\left(\frac{\mu}{T}\right)+\ldots, \\
\delta \mu & =c_{1} \frac{u^{\mu} \partial_{\mu} T}{T}+c_{2} \partial_{\mu} u^{\mu}+c_{3} u^{\mu} \partial_{\mu}\left(\frac{\mu}{T}\right)+\ldots, \\
\delta u^{\mu} & =b_{1} u^{\nu} \partial_{\nu} u^{\mu}+b_{2} \frac{\Delta^{\mu \nu} \partial_{\nu} T}{T}+b_{3} \Delta^{\mu \nu} \partial_{\nu}\left(\frac{\mu}{T}\right)+\ldots
\end{aligned}
$$

The coefficients $\left(a_{i}, b_{i}, c_{i}\right)$ are arbitrary real numbers and the concept of frame is referred to choose some specific values for these numbers. The scalar $\left(\frac{u^{\mu} \partial_{\mu} T}{T}, \partial_{\mu} u^{\mu}, u^{\mu} \partial_{\mu}\left(\frac{\mu}{T}\right)\right)$ and vector $\left(u^{\nu} \partial_{\nu} u^{\mu}, \frac{\Delta^{\mu \nu} \partial_{\nu} T}{T}, \Delta^{\mu \nu} \partial_{\nu}\left(\frac{\mu}{T}\right)\right)$ functions are independent bases and useful to expand 
other quantities. For example, we can express the hydro fields as a function of these bases

$$
\begin{aligned}
& \mathcal{E}=\epsilon_{0}+\sum_{i=1}^{3} \epsilon_{i} s_{i}, \quad \mathcal{P}=p_{\text {id }}+\sum_{i=1}^{3} \pi_{i} s_{i}, \quad \mathcal{N}=n_{0}+\sum_{i=1}^{3} \nu_{i} s_{i}, \\
& \mathcal{Q}^{\mu}=\sum_{i=1}^{3} \theta_{i} v_{i}^{\mu}, \quad \mathcal{J}^{\mu}=\sum_{i=1}^{3} \gamma_{i} v_{i}^{\mu}, \quad t^{\mu \nu}=-\eta \sigma^{\mu \nu}
\end{aligned}
$$

In the latter relations, $s_{i}, v_{i}^{\mu}$ and $\sigma^{\mu \nu}$ are scalar, vector and tensor bases built out of derivatives of thermo fields

$$
\begin{aligned}
& s_{1}=\frac{u^{\mu} \partial_{\mu} T}{T}, \quad s_{2}=\partial \cdot u, \quad s_{3}=u^{\mu} \partial_{\mu}\left(\frac{\mu}{T}\right), \\
& v_{1}^{\mu}=u^{\alpha} \partial_{\alpha} u^{\mu}, \quad v_{2}^{\mu}=\frac{\Delta^{\mu \alpha} \partial_{\alpha} T}{T}, \quad v_{3}^{\mu}=\Delta^{\mu \alpha} \partial_{\alpha}\left(\frac{\mu}{T}\right), \\
& \sigma^{\mu \nu}=\mathcal{P}^{\mu \nu \alpha \beta} \partial_{\alpha} u_{\beta}, \quad \mathcal{P}^{\mu \nu \alpha \beta} \equiv \frac{1}{2}\left(\Delta^{\mu \alpha} \Delta^{\nu \beta}+\Delta^{\mu \beta} \Delta^{\nu \alpha}-\frac{2}{3} \Delta^{\mu \nu} \Delta^{\alpha \beta}\right) .
\end{aligned}
$$

The numbers $\left(\epsilon_{i}, \pi_{i}, \nu_{i}, \theta_{i}, \gamma_{i}, \eta\right)$ are transport coefficients and until no condition is implied, they are arbitrary numbers. Choosing the scalar and vector sets is not unique and one can adopt other sets by just making a linear combination. In the usual use of the $\mathrm{RH}$, people often have benefited from frame freedom defined in the relation (2.9), to fix the frame before any calculation. But the idea of GF tells us that we have to keep the transports undetermined and proceed the computations and after doing them we fix the transports according to our demands. The Difference of my works with respect to the paper [28] is

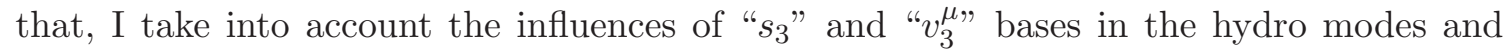
study the stability and causality conditions implied by adding these new bases.

Hereafter, I split the calculations into two parts. The next section is devoted to the calculations of hydro modes for very dense systems $\left(\mu_{0} \gg T_{0}\right)$ and investigating the stability and causality conditions implied on this fluid. Section 4 belongs to the same calculations for finite $\mu_{0}$ and $T_{0}$.

\section{Dense fluids}

Dense fluid is a very cold and charged fluid which has $\mu_{0} \gg T_{0}$ and it does not mean $T=0$, but " $T$ " because of its smallness is treated as a fluctuating field. I have to notify that there is a great difference between the hydrodynamics for a hot and uncharged medium and the hydrodynamics in a cold and dense medium. In the former case, we could safely adopt the following sets of thermodynamics states and fluctuations

$$
\begin{array}{lrrr}
\text { Thermodynamic state, } & \mu_{0}=0, & T_{0} \neq 0, & u_{0}^{\mu}=(1,0,0,0), \\
\text { Fluctuations }, & \delta \mu=0, & \delta T \neq 0, & \delta u^{\mu}=\left(0, \delta u_{x}, \delta u_{y}, \delta u_{z}\right) .
\end{array}
$$

We have four equations (conservation laws of energy-momentum) for four unknown variables $\left(\delta T, \delta u^{x}, \delta u^{y}, \delta u^{z}\right)$ and there is no problem. In the latter case (hydrodynamics of 
a cold and dense medium), we could not set the following thermodynamics states and fluctuations

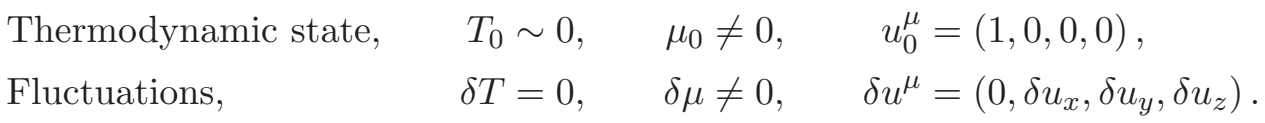

This is because we have five equations (conservation laws of energy-momentum and charge) for four unknown fluctuations $\left(\delta \mu, \delta u^{x}, \delta u^{y}, \delta u^{z}\right)$. Therefore, the hydrodynamic equations of cold and dense medium instead of using the relations (3.3) and (3.4), have started with the following sets

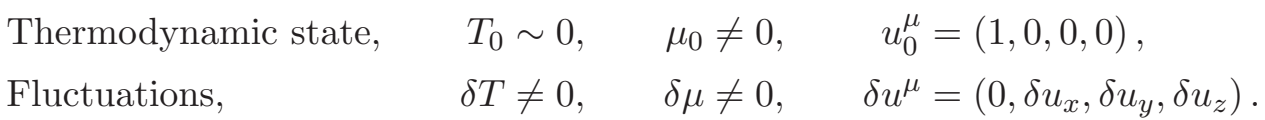

This seems physical, since the chemical potential is only a parameter which adjusts the energy scale to create a charged particle. But, temperature is the more fundamental parameter and thermal fluctuations are related to the motion of particles and they even exist in the very cold medium. The equations (3.5) and (3.6) lead us to the consistent equations which do not need further information. To prove further this argument, recently the $T_{0} \ll \mu_{0}$ limit of fluid/gravity correspondence is studied [32] and it has been shown that by taking some assumptions, we are able to construct the dual fluid of an ADS-RN gravity by solving the Einstein-Maxwell equations, perturbatively. The stability and causality conditions are derived by looking to the hydro modes. I do this work for a fluid at its local rest frame. One can do the same calculation for locally boosted fluids $u^{\mu}=\frac{1}{\sqrt{1-v^{2}}}\left(1, v^{i}\right)$, only by a simple boost transformation [28]. However, having the non zero velocity might cause changing the quality of conditions [26]. I shall show that using the asymptotic causality condition will enable us to obtain correct results for the parameter space of transport coefficients.

To derive the hydro modes, we have to perform some steps. First, we should set our thermodynamic states and fluctuations. I call the thermal fields as $\phi_{a}$ and its fluctuations as $\delta \phi_{a}$

$$
\phi_{a}+\delta \phi_{a}=\left(\delta T, \mu_{0}+\delta \mu, u_{0}^{\mu}+\delta u^{\mu}\right) .
$$

Then, we perturb the constitutive relations up to the first order in fluctuations. In the case of dense fluids, constitutive relations are like as the relations (2.3) and (2.4) in which the hydro fields are written in form of the relations (2.13) and (2.14). Next, we use the conservation laws and solve them. To do this, we write the hydro fluctuations in their Fourier bases

$$
\left(\delta T, \delta \mu, \delta u^{\mu}\right) \rightarrow e^{-i \omega t+i k x}\left(\delta \tilde{T}, \delta \tilde{\mu}, \delta \tilde{u}^{\mu}\right) .
$$

The sign " " refers to the momentum-space version of fluctuations. Rotational invariance permits us to choose the momentum in an arbitrary direction and therefore I select it to be aligned as $k_{\mu}=(\omega, k, 0,0)$. Eventually, the following matrix equation is appeared

$$
\mathcal{M}_{a b} \delta \phi_{b}=0, \quad a, b=1, \cdots 5,
$$


Hydro modes are nothing but the small wave number limit of the following equation

$$
\operatorname{det}[\mathcal{M}]=0 .
$$

Our purpose in the current section is to solve the equation (3.10) for dense fluids. To do this, the sets of scalar and vector bases in hydro fields are chosen as it follows

$$
\begin{array}{lll}
s_{1}=\frac{u^{\alpha} \partial_{\alpha} \mu}{\mu}, & s_{2}=\partial \cdot u, & s_{3}=u^{\alpha} \partial_{\alpha}\left(\frac{T}{\mu}\right), \\
v_{1}^{\mu}=u^{\alpha} \partial_{\alpha} u^{\mu}, & v_{2}^{\mu}=\frac{\Delta^{\mu \alpha} \partial_{\alpha} \mu}{\mu}, & v_{3}^{\mu}=\Delta^{\mu \alpha} \partial_{\alpha}\left(\frac{T}{\mu}\right) .
\end{array}
$$

To analyze better the conditions and throughout this paper, I take the conformal symmetry to be imposed on the theory. Choosing this symmetry is not so accidental, since a dense fluid which has massless particles might has conformal symmetry (if the quantum fluctuations are ignored). In practice, the conformal symmetry constrains the transport parameters [28]. By imposing this symmetry in four dimension, the following relations are appeared

$$
\epsilon_{1}=3 \epsilon_{2}, \quad \epsilon_{i}=3 \pi_{i}, \quad \nu_{1}=3 \nu_{2}, \quad \theta_{1}=\theta_{2}, \quad \gamma_{1}=\gamma_{2} .
$$

Therefore, the number of independent transport parameter has reduced from sixteen to only nine parameters $\left(\epsilon_{1,3}, \nu_{1,3}, \theta_{1,3}, \gamma_{1,3}, \eta\right)$. For the sake of convenience, in the current and next section, I replace the indices " 3 " in parameters by index " 2 " and therefore indices "2" refer to the bases $u^{\alpha} \partial_{\alpha}\left(\frac{\mu}{T}\right)$ and $\Delta^{\mu \alpha} \partial_{\alpha}\left(\frac{\mu}{T}\right)$ or $u^{\alpha} \partial_{\alpha}\left(\frac{T}{\mu}\right)$ and $\Delta^{\mu \alpha} \partial_{\alpha}\left(\frac{T}{\mu}\right)$. By using the bases in the relations (3.11) and (3.12), I repeat all the aforementioned steps for hydro modes. All the linearized equations are collected together to find the matrix $\mathcal{M}_{a b}$. I write the resultant matrix as follows

$$
\begin{aligned}
& \mathcal{M}_{a b} \\
& =\left[\begin{array}{ccccc}
-\frac{\gamma_{1} k^{2}+\omega\left(3 \nu_{1} \omega+i \mu_{0} \chi\right)}{\mu_{0}}-\frac{\gamma_{2} k^{2}+\nu_{2} \omega^{2}}{\mu_{0}} & k\left(i n_{0}+\left(\gamma_{1}+\nu_{1}\right) \omega\right) & 0 & 0 \\
-\frac{\theta_{1} k^{2}+3 \omega\left(\epsilon_{1} \omega+i n_{0} \mu_{0}\right)}{\mu_{0}}-\frac{\theta_{2} k^{2}+\epsilon_{2} \omega^{2}}{\mu_{0}} & k\left(i w_{0}+\left(\epsilon_{1}+\theta_{1}\right) \omega\right) & 0 & 0 \\
\frac{k\left(i n_{0} \mu_{0}+\omega\left(\epsilon_{1}+\theta_{1}\right)\right)}{\mu_{0}} & \frac{k \omega\left(\epsilon_{2}+3 \theta_{2}\right)}{3 \mu_{0}} & -\frac{\left(\epsilon_{1}-4 \eta\right) k^{2}+3 \omega\left(i w_{0}+\theta_{1} \omega\right)}{3} & 0 & 0 \\
0 & 0 & 0 & \eta k^{2}-\omega\left(i w_{0}+\theta_{1} \omega\right) & 0 \\
0 & 0 & 0 & 0 & \eta k^{2}-\omega\left(i w_{0}+\theta_{1} \omega\right)
\end{array}\right] .
\end{aligned}
$$

I use the following thermodynamic relations to derive the latter matrix

$$
\frac{\partial p_{\text {id }}}{\partial \mu}=n_{0}, \quad \frac{\partial n}{\partial \mu}=\chi, \quad w_{0}=\epsilon_{0}+p_{\text {id }}, \quad \frac{\partial \epsilon_{0}}{\partial \mu}=\frac{\partial \epsilon_{0}}{\partial p_{\text {id }}} \frac{\partial p_{\text {id }}}{\partial \mu}=\frac{n_{0}}{c_{s}^{2}}=3 n_{0} .
$$

For the matrix in the relation (3.14), the hydro modes are derived from the solution of the following equations

$$
\begin{gathered}
\operatorname{det}\left[\begin{array}{cc}
\eta k^{2}-\omega\left(i w_{0}+\theta_{1} \omega\right) & 0 \\
0 & \eta k^{2}-\omega\left(i w_{0}+\theta_{1} \omega\right)
\end{array}\right]=\left(\eta k^{2}-\omega\left(i w_{0}+\theta_{1} \omega\right)\right)^{2}=0, \\
\operatorname{det}\left[\begin{array}{ccc}
-\frac{\gamma_{1} k^{2}+\omega\left(3 \nu_{1} \omega+i \mu_{0} \chi\right)}{\mu_{0}} & -\frac{\gamma_{2} k^{2}+\nu_{2} \omega^{2}}{\mu_{0}} & k\left(i n_{0}+\left(\gamma_{1}+\nu_{1}\right) \omega\right) \\
-\frac{\theta_{1} k^{2}+3 \omega\left(\epsilon_{1} \omega+i n_{0} \mu_{0}\right)}{\mu_{0}} & -\frac{\theta_{2} k^{2}+\epsilon_{2} \omega^{2}}{\mu_{0}} & k\left(i w_{0}+\left(\epsilon_{1}+\theta_{1}\right) \omega\right) \\
\frac{k\left(i n_{0} \mu_{0}+\omega\left(\epsilon_{1}+\theta_{1}\right)\right)}{\mu_{0}} & \frac{k \omega\left(\epsilon_{2}+3 \theta_{2}\right)}{3 \mu_{0}} & -\frac{\left(\epsilon_{1}-4 \eta\right) k^{2}+3 \omega\left(i w_{0}+\theta_{1} \omega\right)}{3}
\end{array}\right]=0 .
\end{gathered}
$$



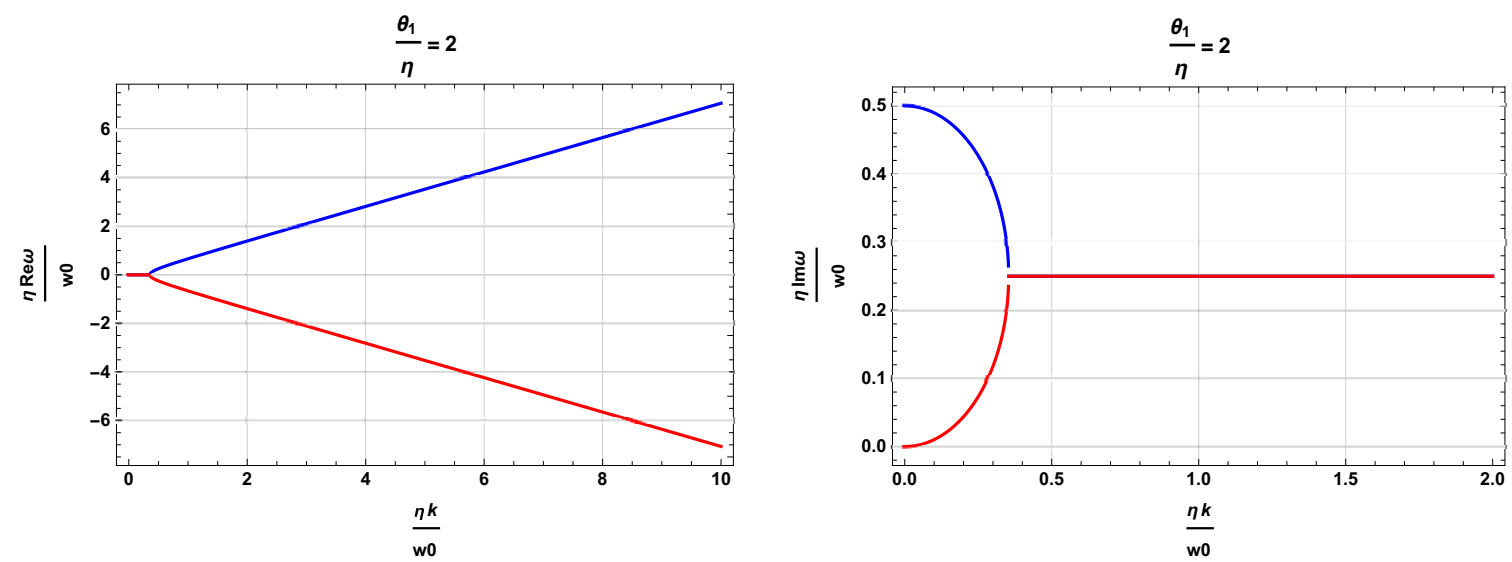

Figure 1. Real and imaginary parts of hydro modes in the shear channel for $\frac{\theta_{1}}{\eta}=2$. Left figure corresponds to the real part and right figure corresponds to the imaginary part of shear modes. The blue and red curves represent two branches of solutions in the relation (3.15).

Splitting the hydro modes into two separate equations has a physical meaning. Solutions of equation (3.16) are known as the shear modes and solutions of equation (3.17) are known as the sound modes. In the following subsections, I shall study these two channels, separately.

\subsection{Shear modes}

Shear channels are denoted by solutions of equation (3.16). The solutions are as

$$
\omega=\frac{w_{0}}{2 \theta_{1}}\left(i \pm \sqrt{\frac{4 \eta \theta_{1} k^{2}}{w_{0}^{2}}-1}\right) .
$$

Hydro modes of this channel are derived from small wavenumber limit of the latter equation

$$
\omega_{\text {hydro }}^{(1)}=\frac{i w_{0}}{\theta_{1}}\left(1-\frac{\eta \theta_{1} k^{2}}{w_{0}^{2}}\right)+\mathcal{O}\left(k^{4}\right), \quad \omega_{\text {hydro }}^{(2)}=\frac{i \eta k^{2}}{w_{0}}+\mathcal{O}\left(k^{4}\right) .
$$

Looking to the equation (3.18) will demonstrate that there is a critical wave number

$$
k_{c}=\frac{w_{0}}{2 \sqrt{\eta \theta_{1}}}
$$

For $k \geq k_{c}$ we have two propagating modes, while for $k<k_{c}$ we have two nonpropagating modes [25]. This is the generic feature of shear modes. According to the relations (3.16) and (3.18), we have four shear modes for charged medium, while in the uncharged case we have only two shear modes. In the figure 1 the real and imaginary parts of the hydro modes are sketched for $\frac{\theta_{1}}{\eta}=2$ and $\frac{\eta k_{c}}{w_{0}}=\frac{1}{2 \sqrt{2}}$.

Stability and causality requirements may constrain the transport parameters $\left(\eta, \theta_{1}\right)$. Stability requires that $\operatorname{Im} \omega \leq 0$ and causality implies that $\left(v_{g}=\frac{\partial R e(\omega)}{\partial k} \leq 1\right)$. One might think that these two concepts are independent of each other, but in the relativistic theory these two issues are correlated to each other. For our case stability gives causality. 
To derive the stability constraints, the $\omega=i \Omega$ has to be inserted into the relation (3.16). Therefore, the stability demands $R e \Omega \leq 0$. This gives rise to the following equation

$$
\Omega^{2} \theta_{1}+\Omega w_{0}+\eta k^{2}=0
$$

Using the Routh-Hurwitz criteria [35], leads to the following conditions

$$
\eta \geq 0, \quad \theta_{1} \geq 0 .
$$

To derive the causality constrains, we look to the asymptotic limit of wave number $(k \rightarrow \infty)$ in the group velocity expression [25]. This statement is verified for MIS theory [25] and we can safely apply it for this general first order hydro. This is because the arguments as explained, are general and do not depend on the detail of theory. For the shear channel solutions given in the relation (3.18), I get the following result for asymptotic velocity

$$
\lim _{k \rightarrow \infty} v_{g}=\sqrt{\frac{\eta}{\theta_{1}}}
$$

Thus, we conclude that causality shrinks the phase space of transport parameters to

$$
\eta \leq \theta_{1}
$$

This constraint is in complete agreement with the condition (29) of the paper [28]. In this paper [28], the author has obtained the equation (3.24) for the locally boosted fluid, while benefiting of asymptotic causality condition [25] has derived the same result without considering the frame velocity. Also, the condition (3.24) can be compared with the similar condition for MIS [25]. Both of these two models (MIS and general first order hydro) have shown that in a causal theory, the shear transport coefficient can not take any arbitrary value and it has an upper bound.

\subsection{Sound modes}

The sound channel is described by the solutions of equation (3.17). The resulting equation is written as it follows

$$
a_{6} \omega^{6}+i a_{5} \omega^{5}+a_{4} \omega^{4}+i a_{3} \omega^{3}+a_{2} \omega^{2}+i a_{1} \omega+a_{0}=0 .
$$

Hydro modes correspond to the small wave number limit of the solutions of latter equation. Expressions of the coefficients $\left(a_{i}, i=1, \ldots 6\right)$ are given in below

$$
\begin{aligned}
a_{6} & \equiv 3 \theta_{1} \mathcal{S}_{1} \\
a_{5} & =\mu_{0}\left(3 n_{0} \mathcal{S}_{1}-\theta_{1} \mathcal{S}_{2}\right) \\
a_{4} & =k^{2}\left(3 \epsilon_{1} \mathcal{S}_{3}-2 \mathcal{S}_{1}\left(2 \eta+\theta_{1}\right)\right)+\mu_{0}^{2} n_{0} \mathcal{S}_{2}, \\
a_{3}= & k^{2} \mu_{0}\left(\frac{\chi}{3}\left(3 \theta_{2} \epsilon_{1}+\epsilon_{2}\left(4 \eta+\theta_{1}\right)\right)\right. \\
& \left.\quad-n_{0}\left(\mathcal{S}_{1}+\epsilon_{2} \ell_{1}+3\left(\theta_{2} \nu_{1}-\mathcal{S}_{3}-\epsilon_{1} \ell_{2}\right)+\nu_{2}\left(4 \eta+\theta_{1}\right)\right)\right),
\end{aligned}
$$




$$
\begin{aligned}
a_{2}= & \frac{k^{2}}{3}\left(k^{2}\left(\theta_{1} \mathcal{S}_{1}+4 \eta\left(\gamma_{1} \epsilon_{2}+3 \theta_{2} \nu_{1}-\theta_{1} \nu_{2}\right)-6 \epsilon_{1}\left(\mathcal{S}_{3}+2 \eta \gamma_{2}\right)\right)\right. \\
& \left.\quad-n_{0} \mu_{0}^{2}\left(\mathcal{S}_{2}+9 \ell_{2} n_{0}\right)\right), \\
a_{1}= & \frac{k^{4} \mu_{0}}{3}\left(\chi \theta_{2}\left(4 \eta-\epsilon_{1}\right)+n_{0}\left(\epsilon_{2} \ell_{1}-3 \epsilon_{1} \ell_{2}+3\left(\theta_{2} \nu_{1}-\mathcal{S}_{3}-4 \eta \gamma_{2}\right)\right)\right), \\
a_{0}= & \frac{k^{4}}{3}\left(k^{2}\left(\epsilon_{1}-4 \eta\right) \mathcal{S}_{3}+3 n_{0}^{2} \mu_{0}^{2} \ell_{2}\right) .
\end{aligned}
$$

In these relations the unknown expressions for $\mathcal{S}_{1,2,3}$ and $\ell_{1,2}$ are defined as

$$
\begin{array}{rlrl}
\mathcal{S}_{1} & \equiv \nu_{2} \epsilon_{1}-\nu_{1} \epsilon_{2}, & \mathcal{S}_{2} \equiv \chi \epsilon_{2}-3 \nu_{2} n_{0}, & \mathcal{S}_{3} \equiv \gamma_{2} \theta_{1}-\gamma_{1} \theta_{2}, \\
\ell_{1,2} & \equiv \gamma_{1,2}-\frac{n_{0}}{w_{0}} \theta_{1,2} .
\end{array}
$$

For dense medium $w_{0}=n_{0} \mu_{0}$ and therefore $\ell_{1,2}=\gamma_{1,2}-\frac{\theta_{1,2}}{\mu_{0}}$. The $a_{i}$ coefficients in the relations (3.26)-(3.32) have featured some properties. The even coefficients $\left(a_{2}, a_{4}, a_{6}\right)$ have even powers of $\mu_{0}$, while the odd coefficients $\left(a_{1}, a_{3}, a_{5}\right)$ have odd powers of $\mu_{0}$. This is not an accidental event. It is such that the charge conjugation symmetry is respected. Also, the even coefficients $a_{2 n}$ are ordered according to the even powers of momentum, while the odd coefficients $a_{2 n-1}$ are proportional to the $k^{6-2 n}$. Another property is that in the even coefficients the greatest powers of momentum (the order $k^{6}$ in $a_{6}$ and so on), have nothing to do with thermodynamics information and just the transport parameters appear, while the next lowest order of momentum have influenced of either the thermodynamics or transport parameters. In the odd coefficients both of the thermodynamics values and transport parameters contribute to expressions.

We are able to derive the variations of $\mathcal{S}_{1,2,3}$ under the fields redefinitions. The expressions $\ell_{1,2}$ are invariant under the thermo field redefinitions. By using the equations (3.15), transformation properties of transport parameters can be recast as follows

$$
\begin{array}{rlrl}
\epsilon_{i} & \rightarrow \epsilon_{i}-3 n_{0} c_{i}, & \nu_{i} & \rightarrow \nu_{i}-\chi c_{i}, \\
\theta_{i} & \rightarrow \theta_{i}-w_{0} b_{i}, & \gamma_{i} \rightarrow \gamma_{i}-n_{0} b_{i}, \\
\eta & \rightarrow \eta . &
\end{array}
$$

In these relations, the $(i=1,2)$ correspond to two sets of transport parameters. Using the latter relations will enable us to derive the transformation properties of $\mathcal{S}_{1,2,3}$. The final result is written as

$$
\begin{aligned}
& \mathcal{S}_{1} \rightarrow \mathcal{S}_{1}-3 n_{0}\left(\nu_{1} c_{2}-\nu_{2} c_{1}\right)-\chi\left(c_{1} \epsilon_{2}-c_{2} \epsilon_{1}\right), \\
& \mathcal{S}_{2} \rightarrow \mathcal{S}_{2}, \\
& \mathcal{S}_{3} \rightarrow \mathcal{S}_{3}-n_{0} \mu_{0}\left(\ell_{2} b_{1}-\ell_{1} b_{2}\right) .
\end{aligned}
$$

Invariance of $\mathcal{S}_{2}$ backs to the EoS of dense fluids $\left(n_{0}=\alpha \mu_{0}^{3}, \chi=3 \alpha \mu_{0}^{2}\right)$ in which $\alpha$ is a positive and real number and depends on the underlying microscopic theory. It is worthwhile to mention that equations (3.38)-(3.40) imply that $a_{i}$ s are not invariant under 
the frame variations. Thus, the stability and causality conditions derived from them, might depend on the chosen frame (special values of $b_{1,2}$ and $c_{1,2}$ ).

I back to examine the symmetries of equation (3.25). Charge conjugation symmetry is an important symmetry which determines the appearance of hydrodynamic equations for particles and antiparticles. If we look to the bases in the relations (3.11) and (3.12), we realize that by charge conjugation transformation $\left(\mu_{0} \rightarrow-\mu_{0}\right)$, the bases transform as

$$
\begin{array}{rlrl}
\left(s_{1}, s_{2}\right) & \rightarrow\left(s_{1}, s_{2}\right), & s_{3} & \rightarrow-s_{3}, \\
\left(v_{1}^{\mu}, v_{2}^{\mu}\right) & \rightarrow\left(v_{1}^{\mu}, v_{2}^{\mu}\right), & v_{3}^{\mu} \rightarrow-v_{3}^{\mu},
\end{array}
$$

On the other hand, energy momentum tensor and current vector transform under the charge conjugation as $\left(T^{\mu \nu}, J^{\mu}\right) \rightarrow\left(T^{\mu \nu},-J^{\mu}\right)$. Therefore, the transport parameters associated with each scalar, vector and tensor bases, change as the

$$
\begin{array}{rlrlrl}
\left(\epsilon_{1}, \epsilon_{2}\right) & \rightarrow\left(\epsilon_{1}, \epsilon_{2}\right), & \epsilon_{3} & \rightarrow-\epsilon_{3}, & \left(\pi_{1}, \pi_{2}\right) \rightarrow\left(\pi_{1}, \pi_{2}\right), & \pi_{3} \rightarrow-\pi_{3}, \\
\left(\nu_{1}, \nu_{2}\right) & \rightarrow-\left(\nu_{1}, \nu_{2}\right), & \nu_{3} \rightarrow \nu_{3}, & & \\
\left(\theta_{1}, \theta_{2}\right) & \rightarrow\left(\theta_{1}, \theta_{2}\right), & \theta_{3} \rightarrow-\theta_{3}, & \left(\gamma_{1}, \gamma_{2}\right) \rightarrow-\left(\gamma_{1}, \gamma_{2}\right), & \gamma_{3} \rightarrow \gamma_{3}, \\
\eta & \rightarrow \eta & & & &
\end{array}
$$

These properties give rise to the following transformations for $\mathcal{S}_{1,2,3}$ and $\ell_{1,2}$

$$
\left(\mathcal{S}_{1}, \mathcal{S}_{3}, \ell_{2}\right) \rightarrow\left(\mathcal{S}_{1}, \mathcal{S}_{3}, \ell_{2}\right), \quad\left(\mathcal{S}_{2}, \ell_{1}\right) \rightarrow-\left(\mathcal{S}_{2}, \ell_{1}\right)
$$

Collecting all these transformations together will result to the invariance of coefficients $\left(a_{i}, i=1, \cdots 6\right)$ under the charge conjugation transformation. Therefore, the equation (3.25) remains invariant under the charge conjugation transformations as it is expected.

Since the sound equation is a sixth order polynomial, its analytical solutions are very subtle to derive. Instead, I study them in special limits. At small wave number limit, the hydro modes can be derived as it follows

$$
\begin{aligned}
\omega_{1} & =-\frac{i \mu_{0} n_{0}}{\theta_{1}}+\mathcal{O}\left(k^{2}\right), \\
\omega_{2} & =\frac{i \mu_{0} \mathcal{S}_{2}}{3 \mathcal{S}_{1}}+\mathcal{O}\left(k^{2}\right), \\
\omega_{3,4} & = \pm \frac{k}{\sqrt{3}}-\frac{2 i k^{2} \eta\left(\mathcal{S}_{2}+3 \chi \theta_{2}-9 n_{0} \gamma_{2}\right)}{3 n_{0} \mu_{0}\left(\mathcal{S}_{2}-9 n_{0} \ell_{2}\right)}+\mathcal{O}\left(k^{3}\right)= \pm \frac{k}{\sqrt{3}}-\frac{2 i k^{2} \eta}{3 n_{0} \mu_{0}}+\mathcal{O}\left(k^{3}\right), \\
\omega_{5,6} & = \pm k \sqrt{\frac{3 n_{0} \ell_{2}}{\mathcal{S}_{2}}}+\frac{3 i k^{2}\left(9 \ell_{2}^{2} n_{0}^{2} a_{5}+3 n_{0} \ell_{2} a_{3} \frac{\mathcal{S}_{2}}{k^{2}}+a_{1} \frac{\mathcal{S}_{2}^{2}}{k^{4}}\right)}{2 \mu_{0}^{2} \mathcal{S}_{2}^{2} n_{0}\left(\mathcal{S}_{2}-9 n_{0} \ell_{2}\right)}+\mathcal{O}\left(k^{3}\right) .
\end{aligned}
$$

In hyperdense fluid the hydro modes will split into four gapless and two gapped modes. The first two modes $\left(\omega_{1,2}\right)$ are nonpropagating modes and indicate the decay of sound modes. They are independent of momentum (at least in the lowest order) and new relaxation times are defined

$$
\tau_{1}=\frac{\theta_{1}}{\mu_{0} n_{0}}, \quad \tau_{2}=-\frac{3 \mathcal{S}_{1}}{\mu_{0} \mathcal{S}_{2}} .
$$


Stability condition constrains the expressions in sound modes. In the " $\omega_{1}$ " channel, I derive $\theta_{1} \geq 0$ which is nothing but the relation (3.22). In the channel $\omega_{2}$, we have

$$
\frac{\mathcal{S}_{2}}{\mathcal{S}_{1}} \leq 0 .
$$

$\mathcal{S}_{2}$ is frame invariant but $\mathcal{S}_{1}$ is not. Further simplification can be done by using the EoS

$$
\frac{\mathcal{S}_{2}}{\mathcal{S}_{1}}=\frac{\epsilon_{2}-\nu_{2} \mu_{0}}{\nu_{2} \epsilon_{1}-\nu_{1} \epsilon_{2}} \leq 0 .
$$

The channels $\omega_{3,4}$ are the familiar sound modes which take the new modification. By using the EoS we can show that

$$
\frac{\mathcal{S}_{2}+3 \chi \theta_{2}-9 n_{0} \gamma_{2}}{\mathcal{S}_{2}-9 n_{0} \ell_{2}}=1 \geq 0 .
$$

In the channel $\omega_{5,6}$ the stability condition requires that

$$
\frac{9 \ell_{2}^{2} n_{0}^{2} a_{5}+3 n_{0} \ell_{2} a_{3} \frac{\mathcal{S}_{2}}{k^{2}}+a_{1} \frac{\mathcal{S}_{2}^{2}}{k^{4}}}{\mathcal{S}_{2}-9 n_{0} \ell_{2}} \leq 0 .
$$

The latter equation can be simplified as

$$
\left(\epsilon_{2}-\nu_{2} \mu_{0}\right) \mathcal{A}-3 \ell_{2} \mu_{0} \mathcal{S}_{1} \leq 0 .
$$

Expression of $\mathcal{A}$ is given in below

$$
\mathcal{A}=\ell_{1} \epsilon_{2}+3\left(\theta_{2} \nu_{1}+\ell_{2} \theta_{1}-\mathcal{S}_{3}-\epsilon_{1} \gamma_{2}\right) .
$$

On the other hand, in this " $\omega_{5,6}$ " channel, the following conditions have to be satisfied

$$
0 \leq \frac{3 n_{0} \ell_{2}}{S_{2}}=\frac{\mu_{0} \ell_{2}}{\epsilon_{2}-\nu_{2} \mu_{0}} \leq 1 .
$$

Both of $\ell_{2}$ and $\mathcal{S}_{2}$ are frame invariant and the latter constraint is a physical constraint independent of frame redifinitions.

We can constrain the transports from causality arguments. As argued before, the asymptotic causality criterion might give the correct result even in the boosted frame. To this purpose, I look to the large wave number limit of sound mode equation (3.25). The $\omega \rightarrow c k$ has to be inserted into the equation (3.25) and pick up only the dominant terms of " $k$ ". After this replacement, the following equation is derived

$$
b_{6} c^{6}+b_{4} c^{4}+b_{2} c^{2}+b_{0}=0 .
$$

This equation has the following solution

$$
c= \pm \sqrt{\frac{2^{\frac{4}{3}} b_{4}^{2}-6 \times 2^{\frac{1}{3}} b_{2} b_{6}-2 b_{4} \mathcal{B}^{\frac{1}{3}}+2^{\frac{2}{3}} \mathcal{B}^{\frac{2}{3}}}{6 b_{6} \mathcal{B}^{\frac{1}{3}}} .}
$$

In the latter relation the $\mathcal{B}$ has the following definition

$$
\begin{aligned}
\mathcal{B} & \equiv \mathcal{C}+\sqrt{\mathcal{C}^{2}-4\left(b_{4}^{2}-3 b_{6} b_{2}\right)}, \\
\mathcal{C} & \equiv 9 b_{2} b_{4} b_{6}-27 b_{0} b_{6}^{2}-2 b_{4}^{3} .
\end{aligned}
$$


The coefficients $\left(b_{6}, b_{4}, b_{2}, b_{0}\right)$ are leading terms of $\left(a_{6}, a_{4}, a_{2}, a_{0}\right)$ in power of momentum $k$

$$
\begin{aligned}
& b_{6}=a_{6}=3 \theta_{1} \mathcal{S}_{1}, \\
& b_{4}=3 \epsilon_{1} \mathcal{S}_{3}-2 \mathcal{S}_{1}\left(2 \eta+\theta_{1}\right), \\
& b_{2}=\frac{\theta_{1} \mathcal{S}_{1}+4 \eta\left(\gamma_{1} \epsilon_{2}+3 \theta_{2} \nu_{1}-\theta_{1} \nu_{2}\right)-6 \epsilon_{1}\left(\mathcal{S}_{3}+2 \eta \gamma_{2}\right)}{3}, \\
& b_{0}=\frac{\mathcal{S}_{3}}{3}\left(\epsilon_{1}-4 \eta\right) .
\end{aligned}
$$

Asymptotic causality condition rules that velocity "c" in the equation (3.61) has to be $c \leq 1$. This condition along with the stability requirements shall strongly constrain the parameter space of transports. In what follows, an example is given to illustrate further such limitation.

The parameter space of conformal dense fluid is nine dimensions. I have not any possibility to constrain all of these nine space dimensions. To analyze better the phase space of transports, I choose specific values for five of them and limit the rest four. These five chosen transports are

$$
\tilde{\epsilon}_{1}=\frac{\epsilon_{1}}{\mu_{0}^{3}}=1, \quad \tilde{\nu}_{1}=\frac{\nu_{1}}{\mu_{0}^{2}}=1, \quad \tilde{\nu}_{2}=\frac{\nu_{2}}{\mu_{0}^{2}}=2, \quad \tilde{\eta}=\frac{\eta}{\mu_{0}^{3}}=1, \quad \tilde{\theta}_{1}=\frac{\theta_{1}}{\mu_{0}^{3}}=2 .
$$

Another transports including $\left(\tilde{\epsilon}_{2}, \tilde{\theta}_{2}, \tilde{\gamma}_{1,2}=\frac{\gamma_{1,2}}{\mu_{0}^{2}}\right)$ are limited according to the stability and causality requirements. The Routh-Hurwitz criteria will limit the coefficients $\left(a_{0}, \cdots a_{6}\right)$ in the relations (3.26)-(3.32) as the following ones

$$
\begin{aligned}
\left(a_{6}, a_{5}, a_{2}, a_{1}\right) & >0, \\
\left(a_{4}, a_{3}, a_{0}\right) & <0 .
\end{aligned}
$$

We have to collect all the stability, causality and Routh-Hurwitz criteria to analyze completely the phase space of transports. The stability conditions in the equations (3.54), (3.57) and (3.59) as well as the Routh-Hurwitz criteria will give us the following non trivial conditions

$$
\begin{array}{cccc}
\tilde{\epsilon}_{2}<2, \quad \tilde{\gamma}_{2}<\tilde{\theta}_{2}, & & \tilde{\gamma}_{2}+2 \geq \tilde{\epsilon}_{2}+\tilde{\theta}_{2}, \\
\text { If } \quad \tilde{\gamma}_{1} \geq 2 \rightarrow \tilde{\epsilon}_{2} \geq-3 \tilde{\theta}_{2}, & \Rightarrow & -\frac{2}{3}<\tilde{\theta}_{2}<0, \\
\text { If } \quad \tilde{\gamma}_{1}<2 \rightarrow \tilde{\epsilon}_{2}<-3 \tilde{\theta}_{2}, \quad \Rightarrow & \tilde{\theta}_{2}>0 .
\end{array}
$$

The equation (3.71) is a definite condition, while the conditions in equations (3.72) and (3.73) depend on our choice. If $\tilde{\gamma}_{1} \geq 2$, the space of transport $\tilde{\theta}_{2}$ is limited to $-\frac{2}{3} \leq \tilde{\theta}_{2} \leq 0$. If $\tilde{\gamma}_{1}<2$, the space of transport $\tilde{\theta}_{2}$ is $\tilde{\theta}_{2} \geq 0$. In the figure 2 these limits on the phase space of $\tilde{\epsilon}_{2}$ and $\tilde{\theta}_{2}$ are shown. These regions are solely derived from Routh-Hurwitz and stability criteria and they have nothing to do with causality.

According to the arguments based on the figure 2, we have to split the phase space of transports into two distinct regions, the $\tilde{\gamma}_{1} \geq 2$ and $\tilde{\gamma}_{1}<2$ region. First, I examine 

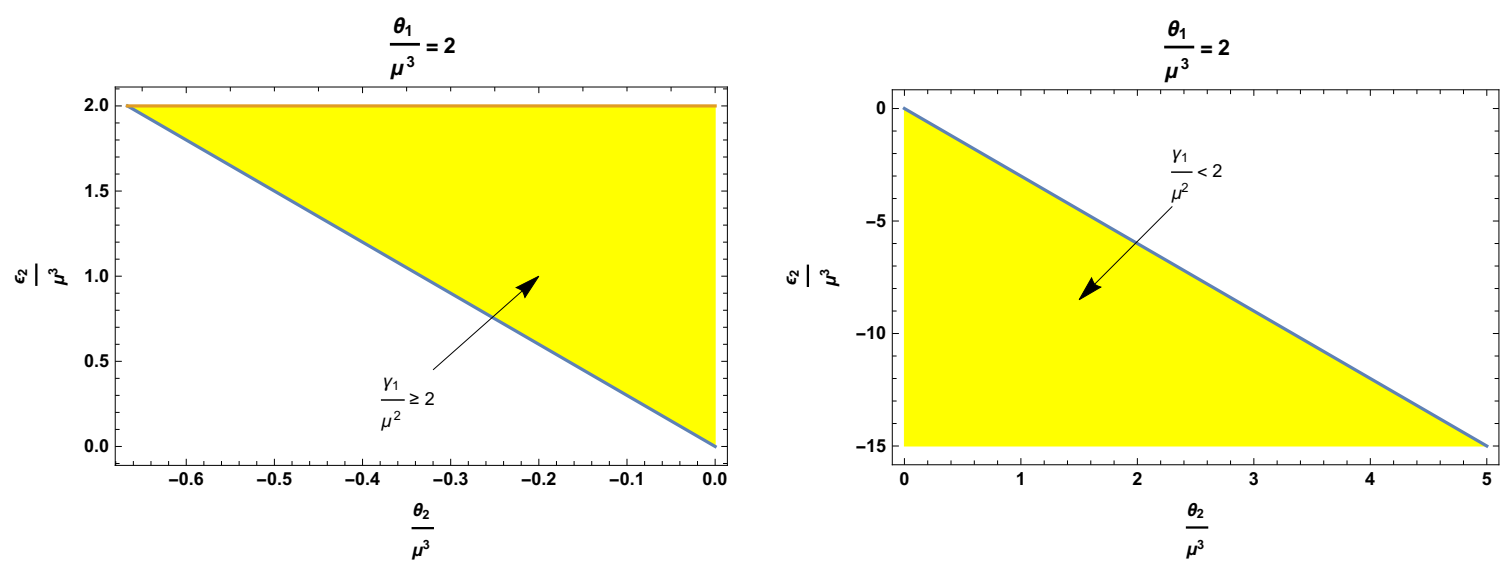

Figure 2. Phase space of transports $\tilde{\epsilon}_{2}$ and $\tilde{\theta}_{2}$ for $\tilde{\theta}_{1}=2$. The left figure corresponds to the region bounded between $\tilde{\epsilon}_{2}<2, \tilde{\epsilon}_{2}=-3 \tilde{\theta}_{2}$ and $\tilde{\theta}_{2}=0$. In this zone $\tilde{\gamma}_{1} \geq 2$ and $-\frac{2}{3}<\tilde{\theta}_{2}<0$. The right figure represents the case $\tilde{\gamma}_{1}<2$ and $\tilde{\theta}_{1}=2$. In this region $\tilde{\epsilon}_{2}<0, \tilde{\theta}_{2}>0$ and $\tilde{\epsilon}_{2}<-3 \tilde{\theta}_{2}$.
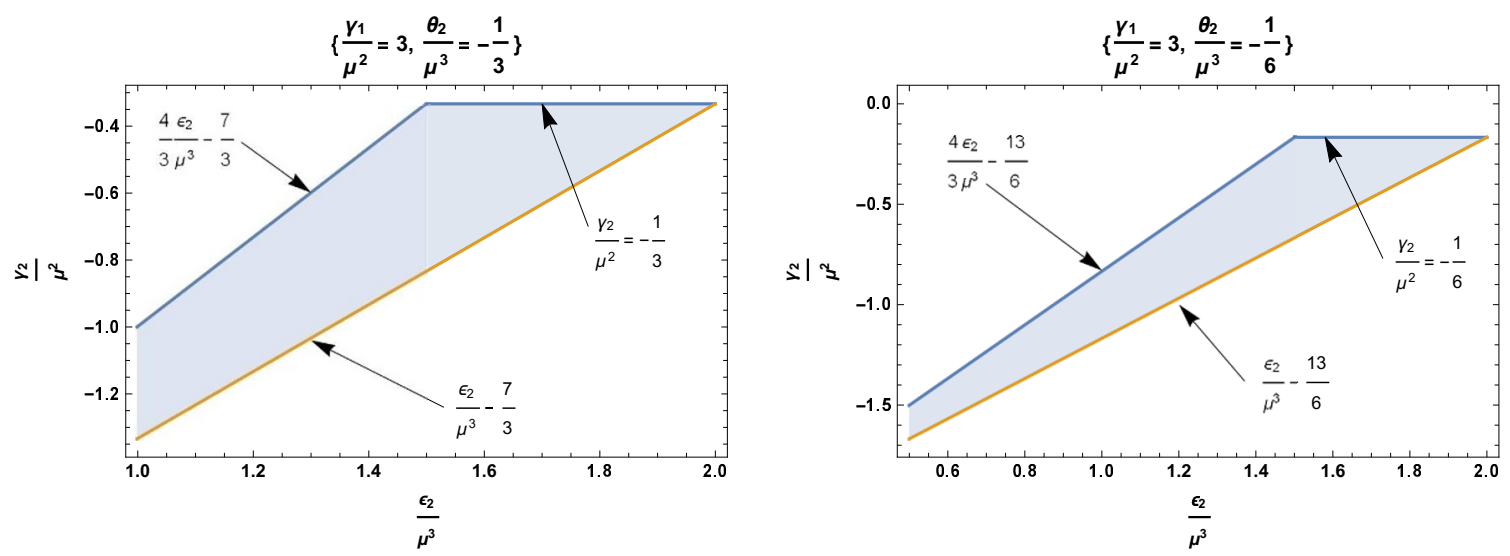

Figure 3. Phase space of transports $\tilde{\gamma}_{2}$ and $\tilde{\epsilon}_{2}$. Both of these plots have $\tilde{\gamma}_{2} \leq 0$ which is compatible with the second law of thermodynamics. Functionality of boundaries are derived from RouthHurwitz and stability criteria. The area of the left and right plot is 0.91 and 1.04, respectively.

the $\tilde{\gamma}_{1} \geq 2$. In the figure 3 the valid regions of $\tilde{\gamma}_{2}$ and $\tilde{\epsilon}_{2}$ for two values $\tilde{\theta}_{2}=-\frac{1}{3}$ and $\tilde{\theta}_{2}=-\frac{1}{6}$ are shown which both of them have $\tilde{\gamma}_{1}=3$. To derive these regions all the RouthHurwitz, stability and causality conditions are put together. The derived conditions from Routh-Hurwitz and stability respect to the causality requirements. The boundary of each diagram is shown in the figure and in both of these plots $\tilde{\gamma}_{2}$ is negative. Functionality of the boundaries are derived from Routh-Hurwitz criteria given in the relations (3.69) and (3.70) and the stability conditions of the relations (3.71). According to the regions shown in the left part of figure 2 as well as the equation (3.72), we must have $1 \leq \tilde{\epsilon}_{2}<2$ for the case $\tilde{\theta}_{2}=-\frac{1}{3}$ and $\frac{1}{2} \leq \tilde{\epsilon}_{2}<2$ for the case $\tilde{\theta}_{2}=-\frac{1}{6}$. The area of left and right plot of figure 3 is 0.91 and 1.04, respectively. Physical meaning of these areas is that what the probability of the respective case is. Therefore, the case $\tilde{\theta}_{2}=-\frac{1}{6}$ is most probable than $\tilde{\theta}_{2}=-\frac{1}{3}$.

In the figure 4 , the permissible zones of $\tilde{\gamma}_{2}$ and $\tilde{\epsilon}_{2}$ for two cases, $\tilde{\gamma}_{1}=5, \tilde{\theta}_{2}=-\frac{1}{3}$ and $\tilde{\gamma}_{1}=5, \tilde{\theta}_{2}=-\frac{1}{6}$ are sketched. We observe that Routh-Hurwitz and stability criteria 

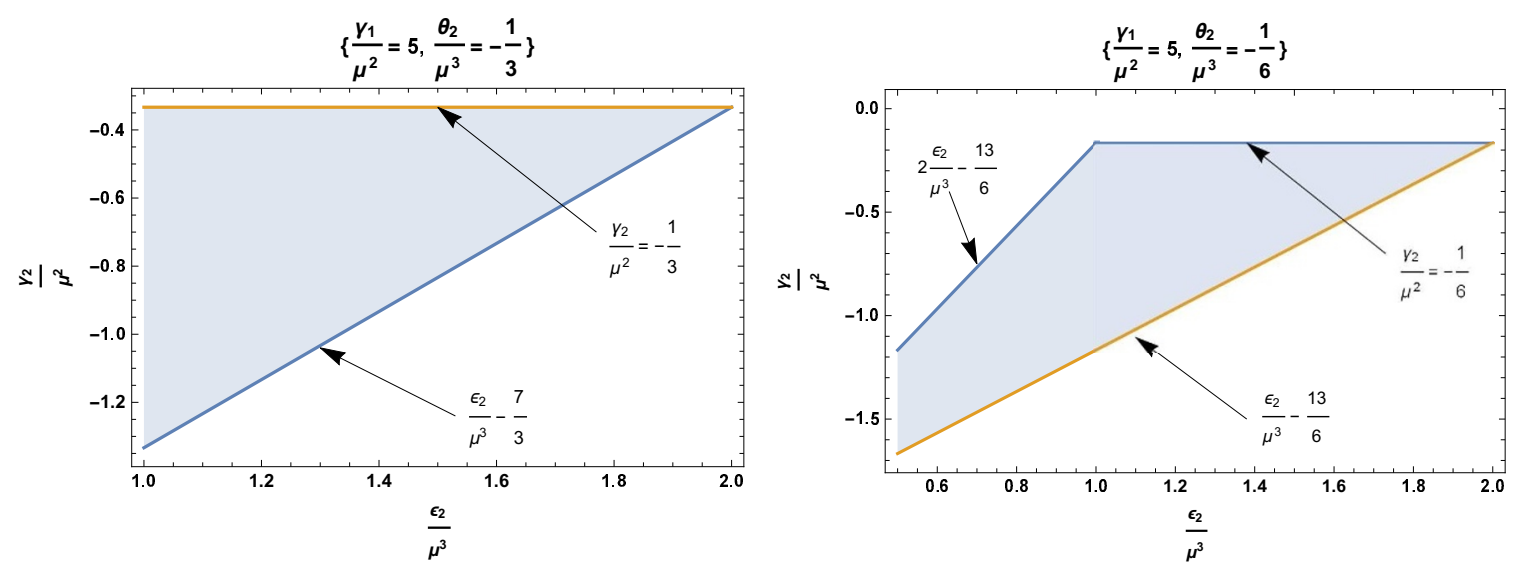

Figure 4. Phase space of transports $\tilde{\gamma}_{2}$ and $\tilde{\epsilon}_{2}$. Both of these plots have $\tilde{\gamma}_{2} \leq 0$ which is compatible with the second law of thermodynamics. Functionality of boundaries are derived from RouthHurwitz and stability criteria. The area of the left and right plot is 0.5 and 0.875 , respectively.

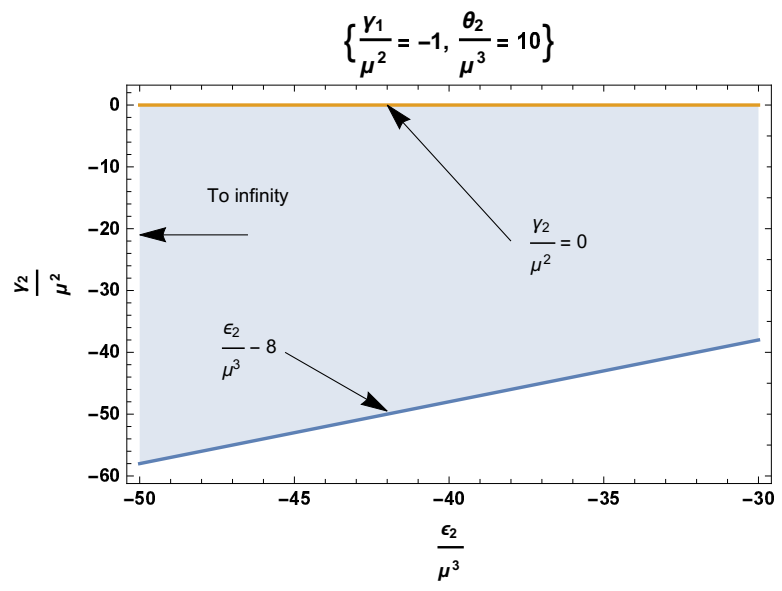

Figure 5. Phase space of transports $\tilde{\gamma}_{2}$ and $\tilde{\epsilon}_{2}$ for $\tilde{\gamma}_{1}=-1$ and $\tilde{\theta}_{2}=10$. Functionality of boundaries are derived from Routh-Hurwitz and stability demands.

support the causality demands. Both of these plots have $\tilde{\gamma}_{2} \leq 0$ and area of left and right part is 0.5 and 0.875 , respectively. I have to mention that for the values of $\tilde{\epsilon}_{2}$ and $\tilde{\theta}_{2}$ sitting on the boundaries in th left part of figure 2 , no acceptable region exist.

Now we go to inspect the $\tilde{\gamma}_{2}<2$ case. Calculations show that for $0<\tilde{\gamma}_{2}<2$, there is no acceptable region compatible with all requirements. For $\tilde{\gamma}_{2}=0$, the acceptable region is only on the line $\tilde{\gamma}_{2}=\frac{\tilde{\epsilon}_{2}}{3}+\tilde{\theta}_{2}-2$, while for $\tilde{\gamma}_{2}<0$, an infinite acceptable region exist. In the figure 5 this zone is shown for $\tilde{\gamma}_{2}=-1$ and $\tilde{\theta}_{2}=10$. The boundaries are derived from Routh-Hurwitz and stability criteria. Stable regions are inside the causal region. If $\tilde{\theta}_{2}$ is increased by fixing the $\tilde{\gamma}$, acceptable region is between the $\tilde{\gamma}_{2}=0$ and $\tilde{\gamma}_{2}=\frac{\tilde{\epsilon}_{2}}{3}+\tilde{\theta}_{2}-2$. From figure 5 , we figure out that the favorable region for $\tilde{\gamma}_{1,2}$ is negative values. For the values of $\tilde{\epsilon}_{2}$ and $\tilde{\theta}_{2}$ sitting on the boundary of right part of figure 2, there is no acceptable region.

We could ask ourselves that do the negative values for transports seem physical or not? The shear and bulk viscosities as well as other (physical) transports are non-negative 
transports. ${ }^{1}$ In the first order general hydro, it has been shown that for uncharged conformal matter, the on-shell solutions in the second law of thermodynamics give rise to the non-negative values for changing transports [28]. However, for charged conformal matter we shall see that negative values for transports are permissible and they do not violate the aforementioned demands. Specially, In the App A. I show that on-shell solutions of conformal charged matter do not rule out the negative values for transports. This is my clear difference with the results of paper [28]. However, this negativeness can be seen not so surprising because transports $\tilde{\gamma}_{1,2} \leq 0$ are not physical ones and they change by frame redefinition. Despite these results, spectral function of corresponding Green function is always positive and second law is respected.

\section{Finite density fluid}

By finite density medium we mean a system which has $\mu_{0} \sim T_{0}$. For this system, the following set of thermodynamic fields and fluctuations is appropriate

$$
\begin{aligned}
& \text { Thermodynamic state, } \quad T_{0}=T_{0}, \quad \mu_{0}=\mu_{0}, \quad u_{0}^{\mu}=(1,0,0,0) \text {, } \\
& \text { Fluctuations, } \quad \delta T \neq 0, \quad \delta \mu \neq 0, \quad \delta u^{\mu}=\left(0, \delta u_{x}, \delta u_{y}, \delta u_{z}\right) \text {. }
\end{aligned}
$$

In this section, the scalar and vector bases are chosen to be as those in relations (2.15)(2.17) and derive the hydro modes by using these bases. Our aim is to constrain the transport parameters and it can be done by studying the hydro modes. The steps given before are repeated and finally the matrix $\mathcal{M}_{a b}$ is derived. Its form is as follows

$\mathcal{M}_{a b}$

$$
=\left[\begin{array}{ccccc}
-\frac{\gamma_{2} k^{2}+i T_{0} \beta_{2} \omega+\nu_{2} \omega^{2}}{T_{0}} & -\frac{k^{2}\left(\gamma_{1} T_{0}-\mu_{0} \gamma_{2}\right)+\omega\left(T_{0}\left(3 \omega \nu_{1}+i T_{0} \beta_{1}\right)-\mu_{0} \omega \nu_{2}\right)}{T_{0}^{2}} & k\left(i n_{0}+\left(\gamma_{1}+\nu_{1}\right) \omega\right) & 0 & 0 \\
-\frac{\theta_{2} k^{2}+\omega\left(\epsilon_{2} \omega+3 i n_{0} T_{0}\right)}{T_{0}}-\frac{k^{2}\left(\theta_{1} T_{0}-\mu_{0} \theta_{2}\right)+\omega\left(3 i T_{0}\left(w_{0}-n_{0} \mu_{0}-i \epsilon_{1} \omega\right)-\mu_{0} \omega \epsilon_{2}\right)}{T_{0}^{2}} & k\left(i w_{0}+\left(\epsilon_{1}+\theta_{1}\right) \omega\right) & 0 & 0 \\
\frac{k\left(3 i n_{0} T_{0}+\omega\left(\epsilon_{2}+3 \theta_{2}\right)\right)}{3 T_{0}} & \frac{k\left(-\mu_{0} \omega\left(\epsilon_{2}+3 \theta_{2}\right)+3 T_{0}\left(i w_{0}-i \mu_{0} n_{0}+\omega\left(\epsilon_{1}+\theta_{1}\right)\right)\right)}{3 T_{0}^{2}} & -\frac{1}{3} k^{2}\left(\epsilon_{1}-4 \eta\right)-\omega\left(i w_{0}+\theta_{1} \omega\right) & 0 & 0 \\
0 & 0 & 0 & \eta k^{2}-\omega\left(i w_{0}+\theta_{1} \omega\right) & 0 \\
0 & 0 & 0 & 0 & \eta k^{2}-\omega\left(i w_{0}+\theta_{1} \omega\right)
\end{array}\right] .
$$

The hydro modes are solutions of following equations

$$
\operatorname{det}\left[\begin{array}{ccc}
-\frac{\gamma_{2} k^{2}+i T_{0} \beta_{2} \omega+\nu_{2} \omega^{2}}{T_{0}} & -\frac{k^{2}\left(\gamma_{1} T_{0}-\mu_{0} \gamma_{2}\right)+\omega\left(T_{0}\left(3 \omega \nu_{1}+i T_{0} \beta_{1}\right)-\mu_{0} \omega \nu_{2}\right)}{T_{0}^{2}} & k\left(i n_{0}+\left(\gamma_{1}+\nu_{1}\right) \omega\right) \\
-\frac{\theta_{2} k^{2}+\omega\left(\epsilon_{2} \omega+3 i n_{0} T_{0}\right)}{T_{0}}-\frac{k^{2}\left(\theta_{1} T_{0}-\mu_{0} \theta_{2}\right)+\omega\left(3 i T_{0}\left(w_{0}-n_{0} \mu_{0}-i \epsilon_{1} \omega\right)-\mu_{0} \omega \epsilon_{2}\right)}{T_{0}^{2}} & k\left(i w_{0}+\left(\epsilon_{1}+\theta_{1}\right) \omega\right) \\
\frac{k\left(3 i n_{0} T_{0}+\omega\left(\epsilon_{2}+3 \theta_{2}\right)\right)}{3 T_{0}} & \frac{k\left(-\mu_{0} \omega\left(\epsilon_{2}+3 \theta_{2}\right)+3 T_{0}\left(i w_{0}-i \mu_{0} n_{0}+\omega\left(\epsilon_{1}+\theta_{1}\right)\right)\right)}{3 T_{0}^{2}} & -\frac{1}{3} k^{2}\left(\epsilon_{1}-4 \eta\right)-\omega\left(i w_{0}+\theta_{1} \omega\right)
\end{array}\right]
$$

The first equation corresponds to the shear modes and the second one corresponds to the sound modes. Shear modes of this section is same as the relation (3.16) and I do not repeat it again. Therefore, I analyze the sound channel.

\footnotetext{
${ }^{1}$ Here, I discuss about theories respect to the parity and therefore I do not consider anomalous transports.
} 


\subsection{Sound channel}

In this channel, the equation (4.5) becomes as

$$
a_{6} \omega^{6}+i a_{5} \omega^{5}+a_{4} \omega^{4}+i a_{3} \omega^{3}+a_{2} \omega^{2}+i a_{1} \omega+a_{0}=0 .
$$

The coefficients $\left(a_{i}, i=1, \cdots 6\right)$ for this case take the following form

$$
\begin{aligned}
a_{6}= & 3 \theta_{1} \mathcal{S}_{1}, \\
a_{5}= & 3\left(w_{0} \mathcal{S}_{1}-\theta_{1} \mathcal{S}_{4}\right) \\
a_{4}= & k^{2}\left(3 \epsilon_{1} \mathcal{S}_{3}-2 \mathcal{S}_{1}\left(2 \eta+\theta_{1}\right)\right)+9 n_{0}^{2} T_{0} \theta_{1}+3 w_{0} \mathcal{S}_{4} \\
a_{3}= & 3 T_{0} w_{0}\left(3 n_{0}^{2}-w_{0} \chi\right)+k^{2}\left(2\left(2 \eta+\theta_{1}\right) \mathcal{S}_{4}\right. \\
a_{2}= & \left.-k^{2}\left(w_{0}\left(\mathcal{S}_{4}+3 w_{0} \epsilon_{2}-3 \gamma_{2} \epsilon_{1}+3 \theta_{2} \nu_{1}-\nu_{2} \theta_{1}+\mathcal{S}_{1}-3 n_{0} \gamma_{1}+\chi\left(4 \eta+\mathcal{S}_{3}\right)\right)\right)+6 n_{0}^{2} T_{0}\left(2 \eta+\theta_{1}\right)\right) \\
& +\frac{k^{4}}{3}\left(\theta_{1} \mathcal{S}_{1}-6 \epsilon_{1} \mathcal{S}_{3}+4 \eta\left(\gamma_{1} \epsilon_{2}-3 \gamma_{2} \epsilon_{1}+3 \theta_{2} \nu_{1}-\theta_{1} \nu_{2}\right)\right) \\
a_{1}= & \frac{k^{4}}{3}\left(w_{0}\left(\epsilon_{2} \ell_{1}-3 \epsilon_{1} \ell_{2}-3\left(\mathcal{S}_{3}+4 \eta \gamma_{2}-\theta_{2} \nu_{1}\right)\right)\right. \\
& \left.\quad-3 n_{0} T_{0}\left(\theta_{1} \nu_{1}-4 \eta \gamma_{2}\right)+\left(\epsilon_{1}-4 \eta\right)\left(\chi \theta_{1} T_{0}-3 n_{0} \theta_{2}\right)\right)-k^{2} T_{0} w_{0}\left(3 n_{0}^{2}-w_{0} \chi\right), \\
a_{0}= & \frac{k^{4}}{3}\left(k^{2}\left(\epsilon_{1}-4 \eta\right) \mathcal{S}_{3}+3 w_{0}^{2} \ell_{2}-3 n_{0} T_{0} w_{0} \ell_{1}\right)
\end{aligned}
$$

Definitions of $\mathcal{S}_{1,3}$ and $\ell_{1,2}$ are same as the relations (3.33) and (3.34). The $\mathcal{S}_{4}$ has the following definition

$$
\mathcal{S}_{4} \equiv \epsilon_{2} n_{0}-w_{0} \nu_{2}+T_{0}\left(3 n_{0} \nu_{1}-\chi \epsilon_{1}\right) .
$$

The following equation is used to derive expressions for $a_{i}$

$$
T_{0}\left(\frac{\partial n_{0}}{\partial T_{0}}\right)_{\mu_{0}}+\mu_{0}\left(\frac{\partial n_{0}}{\partial \mu_{0}}\right)_{T_{0}}=3 n_{0} .
$$

The $a_{i}$ coefficients in the relations (4.7)-(4.13) have the similar features as of the relations (3.26)-(3.32). The sound hydro modes read as

$$
\begin{aligned}
\omega_{1} & =-\frac{i w_{0}}{\theta_{1}}+\mathcal{O}\left(k^{2}\right), \\
\omega_{2,3} & =\frac{i}{2 \mathcal{S}_{1}}\left(\mathcal{S}_{4} \pm \sqrt{\mathcal{S}_{4}^{2}+4 T_{0} \mathcal{S}_{1}\left(3 n_{0}^{2}-w_{0} \chi\right)}\right)+\mathcal{O}\left(k^{2}\right), \\
\omega_{4} & =\frac{i k^{2}\left(n_{0} T_{0} \ell_{1}-w_{0} \ell_{2}\right)}{T_{0}\left(3 n_{0}^{2}-w_{0} \chi\right)}+\mathcal{O}\left(k^{3}\right), \\
\omega_{5,6} & = \pm \frac{k}{\sqrt{3}}-\frac{2 i k^{2} \eta}{3 w_{0}}
\end{aligned}
$$


Unlike the previous section, in the current section the sound mode possess three gapless and three gapped modes. The channels $\omega_{1,2,3}$ are about the decay of sound modes and the corresponding relaxation times are

$$
\tau_{1} \equiv \frac{\theta_{1}}{w_{0}}, \quad \tau_{2,3} \equiv \frac{-2 \mathcal{S}_{1}}{\mathcal{S}_{4} \pm \sqrt{\mathcal{S}_{4}^{2}+4 T_{0} \mathcal{S}_{1}\left(3 n_{0}^{2}-w_{0} \chi\right)}}
$$

Like before, to constrain the transports we have some ways. The Routh-Hurwitz criteria can be imposed independently and the stability demands that

$$
\begin{aligned}
\frac{n_{0} T_{0} \ell_{1}-w_{0} \ell_{2}}{3 n_{0}^{2}-w_{0} \chi} & \leq 0, \\
\frac{\mathcal{S}_{4} \pm \sqrt{\mathcal{S}_{4}^{2}+4 T_{0} \mathcal{S}_{1}\left(3 n_{0}^{2}-w_{0} \chi\right)}}{\mathcal{S}_{1}} & \leq 0 .
\end{aligned}
$$

The causality requirements have to be imposed according to the large momentum limit of group velocity. Since the high momentum limits of even $a_{i}$ s in the expressions (4.7)(4.13) are like as those in the relations (3.26)-(3.32), the asymptotic velocity given in the relation (3.61) can be applied similarly for this case. The only important thing is that how to apply the second law requests. To this purpose, the relation (A.20) is our guide.

To be more concrete, I take the EoS of weakly interacting QGP with $N_{c}$ gluons and $N_{f}$ fermions

$$
\epsilon_{0}=2\left(N_{c}^{2}-1\right) \frac{\pi^{2} T^{4}}{30}+2 N_{f}\left(\frac{7 \pi^{2} T^{4}}{120}+\frac{\mu^{2} T^{2}}{4}+\frac{\mu^{4}}{8 \pi^{2}}\right) .
$$

In what follows, I take $N_{c}=N_{f}=3$ and split the analysis into two cases: 1 ) $x=\frac{\mu_{0}}{T_{0}}=1.30$ and 2) $x=\frac{\mu_{0}}{T_{0}}=0.23$. These values are taken so that $\frac{4 \epsilon_{0}}{3 n_{0} T_{0}}$ becomes 50 and 10 , respectively. Routh-Hurwitz and stability requirements give us two conditions independent of $x$

$$
\tilde{\gamma}_{2}>r\left[\tilde{\gamma}_{1}, \tilde{\theta}_{2}\right]=\frac{\tilde{\gamma}_{1} \tilde{\theta}_{2}}{2}, \quad 2 \tilde{\epsilon}_{2} \tilde{\gamma}_{1}+3\left(2+\tilde{\gamma}_{1}\right) \tilde{\theta}_{2} \geq 6+\tilde{\epsilon}_{2}+12 \tilde{\gamma}_{2}
$$

Unlike the previous section, in this section scale the transports in terms of $T_{0}$

$$
\tilde{\epsilon}_{1,2}=\frac{\epsilon_{1,2}}{T_{0}^{3}}, \quad \tilde{\theta}_{1,2}=\frac{\theta_{1,2}}{T_{0}^{3}}, \quad \tilde{\eta}=\frac{\eta}{T_{0}^{3}}, \quad \tilde{\gamma}_{1,2}=\frac{\gamma_{1,2}}{T_{0}^{2}}, \quad \tilde{\nu}_{1,2}=\frac{\nu_{1,2}}{T_{0}^{2}}
$$

For two above mentioned values of $x$, I list the $x$ dependent constraints in table 1 which are derived from stability, causality and second law requirements. To compare better the analysis with those of dense fluid, I choose the same values for $\left(\tilde{\epsilon}_{1,2}, \tilde{\nu}_{1}, \tilde{\theta}_{1}, \tilde{\eta}\right)$ as ones in relation (3.68) and values of $\tilde{\gamma}_{1}$ and $\tilde{\theta}_{2}$ are taken as before. Like the previous section, the plots are studied in two distinct branches, including the branch of $\tilde{\gamma}_{1} \geq 2$ and $\tilde{\gamma}_{1}<2$.

For the first branch, I take four plots with $\left(\tilde{\gamma}_{1}=3, \tilde{\theta}_{2}=-\frac{1}{3}\right),\left(\tilde{\gamma}_{1}=3, \tilde{\theta}_{2}=-\frac{1}{6}\right)$, $\left(\tilde{\gamma}_{1}=5, \tilde{\theta}_{2}=-\frac{1}{3}\right)$ and $\left(\tilde{\gamma}_{1}=5, \tilde{\theta}_{2}=-\frac{1}{6}\right)$ each of which has $x=1.3$ and $x=0.23$. In the figure 6 , the acceptable zones for $x=1.3$ and $x=0.23$ with the sets $\left(\tilde{\gamma}_{1}=3, \tilde{\theta}_{2}=-\frac{1}{3}\right)$, 


\begin{tabular}{|c|c|}
\hline$x=1.3$ & $x=0.23$ \\
\hline$\tilde{\gamma}_{2} \leq C_{1}\left[\tilde{\gamma}_{1}, \tilde{\theta}_{2}\right]=\tilde{\theta}_{2}+0.1 \tilde{\gamma}_{1}-0.2$ & $\tilde{\gamma}_{2} \leq D_{1}\left[\tilde{\gamma}_{1}, \tilde{\theta}_{2}\right]=\tilde{\theta}_{2}+0.02 \tilde{\gamma}_{1}-0.04$ \\
\hline$\tilde{\gamma}_{2} \leq C_{2}\left[\tilde{\gamma}_{1}, \tilde{\theta}_{2}\right]=0.1\left(\tilde{\gamma}_{1}+\tilde{\theta}_{2}\right)-0.02$ & $\tilde{\gamma}_{2} \leq D_{2}\left[\tilde{\gamma}_{1}, \tilde{\theta}_{2}\right]=0.02\left(\tilde{\gamma}_{1}+\tilde{\theta}_{2}\right)-0.0008$ \\
\hline$\tilde{\gamma}_{2} \leq C_{3}\left[\tilde{\epsilon}_{2}, \tilde{\gamma}_{1}, \tilde{\theta}_{2}\right]=1.37+\tilde{\epsilon}_{2}\left(0.11 \tilde{\gamma}_{1}-0.2\right)+\frac{\tilde{\theta}_{2}}{3}\left(1+\tilde{\gamma}_{1}\right)$ & $\tilde{\gamma}_{2} \leq D_{3}\left[\tilde{\epsilon}_{2}, \tilde{\gamma}_{1}, \tilde{\theta}_{2}\right]=1.57+\tilde{\epsilon}_{2}\left(0.11 \tilde{\gamma}_{1}-0.12\right)+\frac{\tilde{\theta}_{2}}{3}\left(1+\tilde{\gamma}_{1}\right)$ \\
\hline$\tilde{\gamma}_{2} \leq C_{4}\left[\tilde{\epsilon}_{2}, \tilde{\gamma}_{1}, \tilde{\theta}_{2}\right]=0.71-0.03 \tilde{\epsilon}_{2}+0.1 \tilde{\gamma}_{1}+\tilde{\theta}_{2}$ & $\tilde{\gamma}_{2} \leq D_{4}\left[\tilde{\epsilon}_{2}, \tilde{\gamma}_{1}, \tilde{\theta}_{2}\right]=0.84-0.006 \tilde{\epsilon}_{2}+0.2 \tilde{\gamma}_{1}+\tilde{\theta}_{2}$ \\
\hline$\tilde{\gamma}_{2} \leq C_{5}\left[\tilde{\epsilon}_{2}, \tilde{\gamma}_{1}, \tilde{\theta}_{2}\right]=-0.06+\tilde{\epsilon}_{2}\left(0.05 \tilde{\gamma}_{1}-0.1\right)+\tilde{\theta}_{2}\left(0.15 \tilde{\gamma}_{1}+0.34\right)$ & $\tilde{\gamma}_{2} \leq D_{5}\left[\tilde{\epsilon}_{2}, \tilde{\gamma}_{1}, \tilde{\theta}_{2}\right]=-0.03+\tilde{\epsilon}_{2}\left(0.048 \tilde{\gamma}_{1}-0.096\right)+\tilde{\theta}_{2}\left(0.14 \tilde{\gamma}_{1}+0.29\right)$ \\
\hline
\end{tabular}

Table 1. Lists of constrains for two values of $x=\frac{\mu_{0}}{T_{0}}$, including the $x=1.3$ in the left and $x=0.23$ in the right column, stemming from the Routh-Hurwitz, stability and second law criteria.
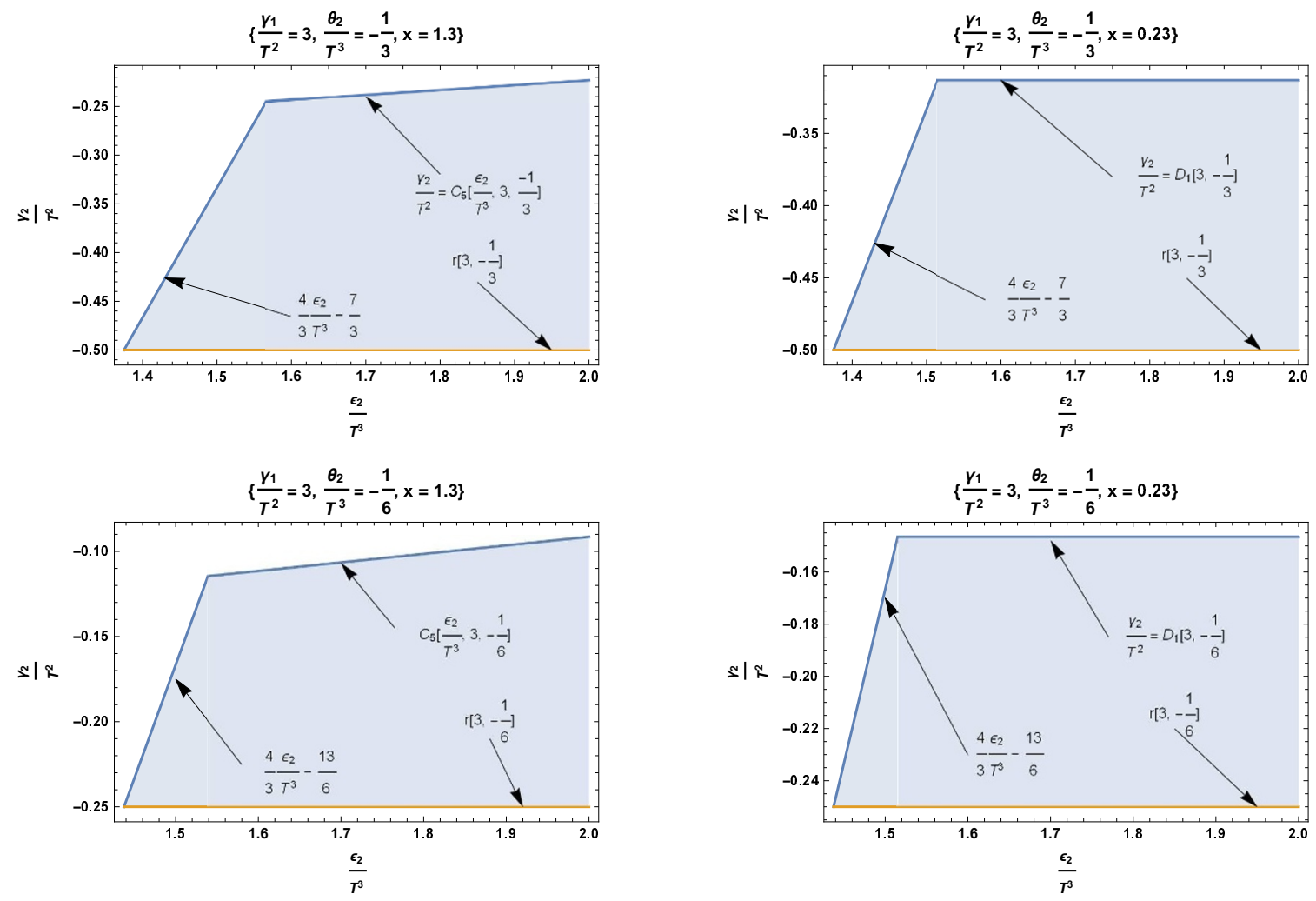

Figure 6. Acceptable zones in the case of finite $\mu_{0}$ and $T_{0}$. Boundaries are given in terms of table 1 data. From top-left to bottom right figure the areas are 0.14, 0.10, 0.07 and 0.05, respectively.

$\left(\tilde{\gamma}_{1}=3, \tilde{\theta}_{2}=-\frac{1}{6}\right)$ is shown. The boundaries of each plot is shown in terms of the corresponding conditions given in the table 1 and the relation (4.25). Areas of the plots from top-left to bottom-right are $(0.14,0.10,0.07,0.05)$, horizontally. Values of transports lie inside these zones do not contradict the asymptotic causality condition. Compared to the similar plots of figure 3 , having a finite ratio for $x$ decrease the accessible zone. If $x \rightarrow 0$, the acceptable zone shrinks more and more and thus the high density medium is much more favorable for these values of $\tilde{\gamma}_{1}$ and $\tilde{\theta}_{2}$. In the figure 7 , the same plots are shown for $x=1.3$ and $x=0.23$ with $\left(\tilde{\gamma}_{1}=5, \tilde{\theta}_{2}=-\frac{1}{3}\right),\left(\tilde{\gamma}_{1}=5, \tilde{\theta}_{2}=-\frac{1}{6}\right)$. The areas of the plots from top-left to bottom-right are $(0.69,0.61,0.41,0.32)$, horizontally. These physical zones already satisfy the asymptotic causality condition. Compared to the similar plots in the figure 4 , having a finite ratio for $x$ increase the accessible zone, while for $\left(\tilde{\gamma}_{1}=5, \tilde{\theta}_{2}=-\frac{1}{6}\right)$ 

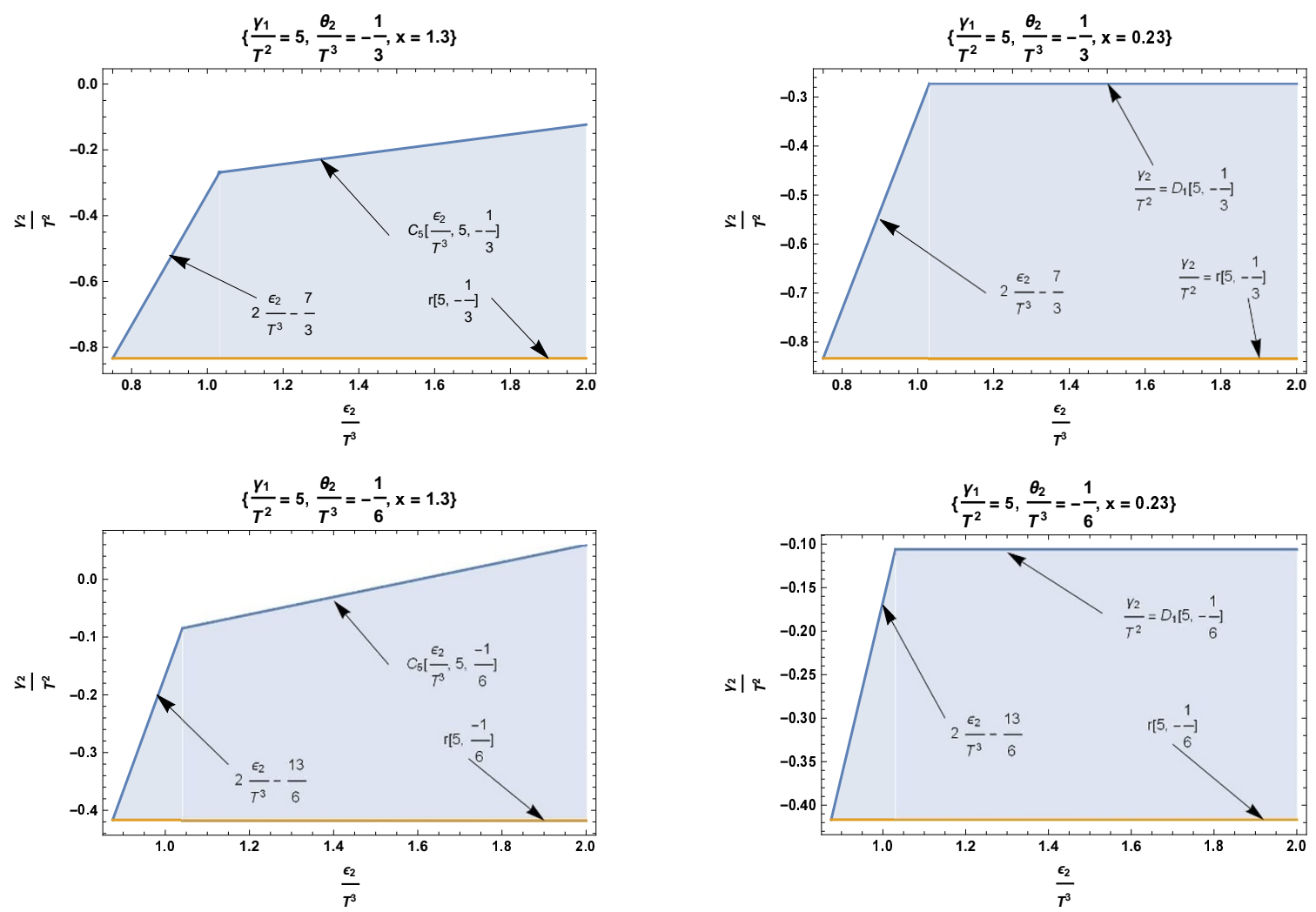

Figure 7. Acceptable zones in the case of finite $\mu_{0}$ and $T_{0}$. Boundaries are given terms of table 1 data. From top-left to bottom-right figure the area of plot is $0.69,0.61,0.41$ and 0.32 , respectively.

a decreasing pattern is seen. For the values $\tilde{\epsilon}_{2}$ and $\tilde{\theta}_{2}$ sitting on the boundaries of left part of figure 2 , there is no acceptable region.

If $0<\tilde{\gamma}_{1}<2$ there is no accessible zone. For $\tilde{\gamma}_{1}=0$ until a critical value of $\tilde{\theta}_{2}$ isn't meet, we have no physical zone, but after it a large area appears. In the figure 8 , I show this area for $\left(\tilde{\gamma}_{1}=0, \tilde{\theta}_{2}=10\right)$ for each of the $x$ values. Compared to dense medium, the physical spaces for transport becomes larger which shows that for $\tilde{\gamma}_{1}=0$ the finite density medium is the most favorable case. To remind again, in dense medium for $\tilde{\gamma}_{1}=0$, the valid space is on the line $\tilde{\gamma}_{2}=\frac{\tilde{\epsilon}_{2}}{3}+\tilde{\theta}_{2}-2$.

If $\tilde{\gamma}_{1}<0$, until a critical value of $\tilde{\theta}_{2}$ isn't meet, there is no valid zone, while after it an infinite physical space emerges. In the figure 9 , this zone is shown for $\left(\tilde{\gamma}_{1}=-1, \tilde{\theta}_{2}=10\right)$ for each of the $x$ values. Unlike the all previous cases, in the finite $x$ with $\tilde{\gamma}_{1}<0$ and after the critical $\tilde{\theta}_{2}$, the physical zone has infinite area. Similar case for dense medium is shown in the figure 5 which has infinite area. It seems that the space $\tilde{\gamma}_{2}<0$ is the most favorable region for finite density medium.

\section{Conclusion}

Stability and causality problems in dissipative hydrodynamics is one of the long standing challenges in the RH. Historically, this problem is remedied by adding a phenomenological 

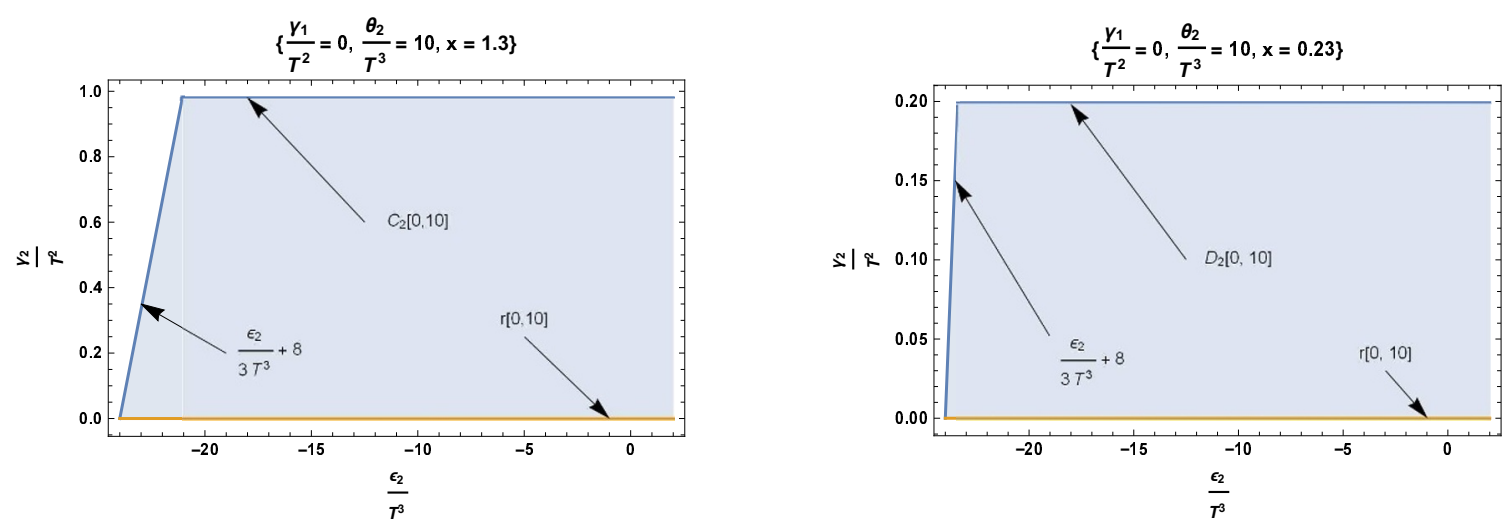

Figure 8. Acceptable zones in the case of finite $\mu_{0}$ and $T_{0}$. Boundaries are given in terms of table 1 data. The left and right figure has area of 24.04 and 5.11, respectively.
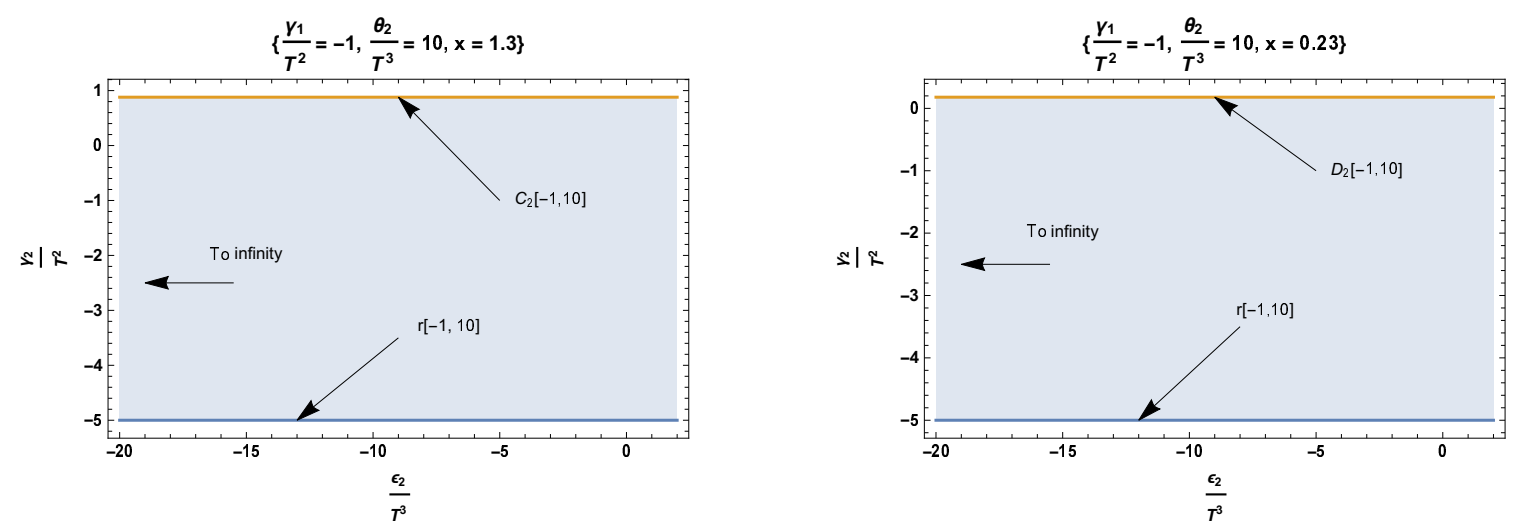

Figure 9. Acceptable zones in the case of finite $\mu_{0}$ and $T_{0}$. Boundaries are given in terms of table 1 data. For $\tilde{\gamma}_{1}<0$ the accessible zone has infinite area which shows the favorablity of this value.

equation to the known conservation equation and treating the dissipative tensors as new DoF. This strategy suffers from lacking the fundamental bases and has no physical background. The newly developed GF notion has resolved the stability and causality problems without introducing artificial terms. This idea has benefited from the frame concept in dissipative hydrodynamic and does not fix it before studying the physical conditions. Indeed, the correct hydrodynamic frame in the view of the GF is a frame in which respects to all physical and high energetic conditions.

This work tries to give the physical and acceptable region of transports for dense medium. I adopt the conformal charged matter in order to analyze better the conditions. Charge conjugation symmetry implies that the $\mathrm{RH}$ equations for particles and antiparticles are identical. The main achievement of this paper is that for conformal charged matter, the second law of thermodynamics $\left(\partial_{\mu} S^{\mu} \geq 0\right)$ has not ruled out the existence of negative transports. Existence of negative transports is illustrated theoretically and numerically. The sign of scalar transports for conformal matter in the GF framework are not limited by any constraints, but sign of the vector transports combination is limited by using the second law and in extreme limits such as $x \rightarrow 0$ or $x \rightarrow \infty$, the second law definitely 
tells that $\tilde{\gamma}_{2} \leq 0$. These negative values are benchmarks of charged fluid, since in the uncharged fluid case [28], the changing transports never acquire negative values. By fixing some transports, the good regions for other transports including the $\tilde{\gamma}_{2}$ and $\tilde{\epsilon}_{2}$ are derived. In the case of finite $T_{0}$ and $\mu_{0}$, the work is done for two ratios of $(x=1.3,0.23)$ and the conditions for each of these $x$ is derived and tabulated. Areas of plot are good judges for the favorability of each case. The conditions derived from Routh-Hurwitz, stability and second law constraints, have respected to the asymptotic causality condition.

The GF framework is in its infant age and deserves a lot attention and we could extend it in various lines. We can construct a microscopic relation for these transports, i.e. the Green-Kubo formalism or other relations and observe the fingerprints of this new transports on the microscopic theory evolution. Other important question is that to construct a holographic picture for the GF framework. Also there are some problems concerning the GF framework [14] which necessitate the use of higher order terms in the GF constitutive relations.

\section{Acknowledgments}

I would like to thank the M. Shokri because of fruitful discussions and comments. I also appreciate the U. Heinz to encourage me to do this work.

\section{A On-shell entropy and negativeness of transports}

For canonical entropy current of a charged fluid following definition is used

$$
T S_{\text {can }}^{\mu}=p_{\text {id }} u^{\mu}-T^{\mu \nu} u_{\nu}-\mu J^{\mu} .
$$

This form of entropy is invariant under the frame redefinition [4] and the index "id" refers to the ideal part of the pressure. Plugging the corresponding expressions for energy momentum tensor and vector current will reach us to the following result

$$
T S_{\text {can }}^{\mu}=\left(T s_{\text {id }}+\mathcal{E}_{\text {res }}-\mu \mathcal{N}_{\text {res }}\right) u^{\mu}+\mathcal{Q}^{\mu}-\mu \mathcal{J}^{\mu} .
$$

$\mathcal{E}_{\text {res }}$ and $\mathcal{N}_{\text {res }}$ are the resistive parts of energy and number density given in the relations (2.13) and (2.14) and $T s_{\text {id }}=\epsilon_{0}+p_{\text {id }}-\mu n_{0}$. By using the EoM, divergence of canonical entropy can be written as

$$
\partial_{\mu} S_{\text {can }}^{\mu}=-T_{\text {res }}^{\mu \nu} \partial_{\nu}\left(\frac{u_{\nu}}{T}\right)-\mathcal{J}^{\mu} \partial_{\mu}\left(\frac{\mu}{T}\right) .
$$

For charged conformal matter thanks to the relation (3.13), after a little computation the following off-shell relation is derived

$$
\partial_{\mu} S_{\text {can }}^{\mu}=S^{T} \cdot M_{s} \cdot S+V_{\mu}^{T} \cdot M_{v} \cdot V^{\mu} .
$$

The "T" stands for transpose and $S$ and $V$ are scalar and vector bases

$$
\begin{aligned}
S & =\left(\frac{u^{\mu} \partial_{\mu} T}{T}, \partial_{\mu} u^{\mu}, u^{\mu} \partial_{\mu}\left(\frac{\mu}{T}\right)\right)^{T}, \\
V^{\mu} & =\left(u^{\nu} \partial_{\nu} u^{\mu}, \frac{\Delta^{\mu \nu} \partial_{\nu} T}{T}, \Delta^{\mu \nu} \partial_{\nu}\left(\frac{\mu}{T}\right)\right)^{T} .
\end{aligned}
$$


$M_{s}$ and $M_{v}$ are scalar and vector matrices involve the transports

$$
\begin{aligned}
M_{s}= & \left(\begin{array}{ccc}
-3 \epsilon_{1} & -\epsilon_{1} & -\frac{3 \nu_{1}+\epsilon_{2}}{2} \\
-\epsilon_{1} & -\frac{\epsilon_{1}}{3} & -\frac{\nu_{1}}{2}-\frac{\epsilon_{2}}{6} \\
-\frac{3 \nu_{1}+\epsilon_{2}}{2} & -\frac{\nu_{1}}{2}-\frac{\epsilon_{2}}{6} & -\nu_{2}
\end{array}\right), \\
M_{v}= & \left(\begin{array}{ccc}
-\theta_{1} & -\theta_{1} & -\frac{\gamma_{1}+\theta_{2}}{2} \\
-\theta_{1} & -\theta_{1} & -\frac{\gamma_{1}+\theta_{2}}{2} \\
-\frac{\gamma_{1}+\theta_{2}}{2} & -\frac{\gamma_{1}+\theta_{2}}{2} & -\gamma_{2}
\end{array}\right) .
\end{aligned}
$$

The transports on this level (off-shell level) have not to be limited, since transports are frame dependent quantities while the entropy current is a frame independent one. Therefore, the relation (A.4) has to be studied in the on-shell limit by using the EoM. Following scalar equations for charged fluid is used taking the equilibrium values for thermo fields

$$
\begin{aligned}
u^{\mu} \partial_{\mu} \epsilon_{0}+\left(\epsilon_{0}+p_{\text {id }}\right) \partial_{\mu} u^{\mu} & =0, \\
u^{\mu} \partial_{\mu} n_{0}+n_{0} \partial_{\mu} u^{\mu} & =0
\end{aligned}
$$

to vanish two of scalar bases in favor of another one. The EoS for conformal charged matter is taken as below

$$
\begin{aligned}
& \frac{\epsilon_{0}}{T^{4}}=\frac{3 p_{\mathrm{id}}}{T^{4}}=a+b\left(\frac{\mu}{T}\right)^{2}+c\left(\frac{\mu}{T}\right)^{4}, \\
& \frac{n_{0}}{T^{3}}=\frac{1}{3 T^{3}} \frac{\partial p_{\mathrm{id}}}{\partial \mu}=\frac{2}{3}\left(2 c\left(\frac{\mu}{T}\right)^{3}+b\left(\frac{\mu}{T}\right)\right), \\
& \frac{s_{\mathrm{id}}}{T^{3}}=\frac{1}{3 T^{3}} \frac{\partial p_{\mathrm{id}}}{\partial T}=\frac{2}{3}\left(2 a+b\left(\frac{\mu}{T}\right)^{2}\right) .
\end{aligned}
$$

The latter relations have to be inserted into the relations (A.9) and (A.10) and the following equations are obtained

$$
\begin{aligned}
\epsilon_{0} \frac{u^{\mu} \partial_{\mu} T}{T}+\frac{3 n_{0} T}{4} u^{\mu} \partial_{\mu}\left(\frac{\mu}{T}\right) & =-\frac{\partial_{\mu} u^{\mu}}{3} \epsilon_{0}, \\
n_{0} \frac{u^{\mu} \partial_{\mu} T}{T}+\chi T u^{\mu} \partial_{\mu}\left(\frac{\mu}{T}\right) & =-\partial_{\mu} u^{\mu} n_{0} .
\end{aligned}
$$

Solutions of equations (A.14) and (A.15) are very simple

$$
\frac{u^{\mu} \partial_{\mu} T}{T}=-\frac{\partial_{\mu} u^{\mu}}{3}, \quad u^{\mu} \partial_{\mu}\left(\frac{\mu}{T}\right)=0 .
$$

Same work can be done for vector bases by using the following equation

$$
u^{\nu} \partial_{\nu} u^{\mu}=-\frac{\Delta^{\mu \nu} \partial_{\nu} p_{\text {id }}}{\epsilon_{0}+p_{\text {id }}} .
$$

The EoS as in the relations (A.11) to (A.13) has to be plugged into the latter relation and finally we get

$$
-u^{\nu} \partial_{\nu} u^{\mu}=\frac{\Delta^{\mu \nu} \partial_{\nu} T}{T}+\frac{3 n_{0} T}{4 \epsilon_{0}} \Delta^{\mu \nu} \partial_{\nu}\left(\frac{\mu}{T}\right) .
$$


By this relation, $\Delta^{\mu \nu} \partial_{\nu}\left(\frac{\mu}{T}\right)$ has to be eliminated in favor another two. Eventually, equation (A.16) along with relation (A.18) are used to make on-shell the second law expression

$$
\partial_{\mu} S_{\text {can }}^{\mu}=\left(u^{\nu} \partial_{\nu} u^{\mu}+\frac{\Delta^{\mu \nu} \partial_{\nu} T}{T}\right)^{2}\left(-\left(\frac{4 \epsilon_{0}}{3 n_{0} T}\right)^{2} \gamma_{2}+\frac{4 \epsilon_{0}}{3 n_{0} T}\left(\gamma_{1}+\theta_{2}\right)-\theta_{1}\right) .
$$

As it is evident, the scalar sections do not enter into the second law and only the vector transports can be studied. This is a weird result and it is because of the conformal symmetry. In order to satisfy the second law demand $\partial_{\mu} S^{\mu} \geq 0$, we must have

$$
-\left(\frac{4 \epsilon_{0}}{3 n_{0} T}\right)^{2} \gamma_{2}+\frac{4 \epsilon_{0}}{3 n_{0} T}\left(\gamma_{1}+\theta_{2}\right)-\theta_{1} \geq 0 .
$$

In this way the vector transports are not limited individually, but the combination of them is limited. For example in the high density medium $(\mu \gg T)$, which $\frac{4 \epsilon_{0}}{3 n_{0} T} \rightarrow \infty$ we have $\gamma_{2} \leq 0$. In the high temperature limit the similar event happens. Thus, in extreme limits we deal with negative transport $\gamma_{2}$ and they do not violate the second law of thermodynamics.

Open Access. This article is distributed under the terms of the Creative Commons Attribution License (CC-BY 4.0), which permits any use, distribution and reproduction in any medium, provided the original author(s) and source are credited.

\section{References}

[1] P. Romatschke and U. Romatschke, Relativistic fluid dynamics in and out of equilibrium, Cambridge University Press, Cambridge, U.K. (2019) [arXiv:1712.05815] [INSPIRE].

[2] W. Florkowski, M.P. Heller and M. Spalinski, New theories of relativistic hydrodynamics in the LHC era, Rept. Prog. Phys. 81 (2018) 046001 [arXiv: 1707.02282] [InSPIRE].

[3] S. Jeon and U. Heinz, Introduction to hydrodynamics, Int. J. Mod. Phys. E 24 (2015) 1530010 [arXiv:1503.03931] [InSPIRE].

[4] P. Kovtun, Lectures on hydrodynamic fluctuations in relativistic theories, J. Phys. A 45 (2012) 473001 [arXiv:1205.5040] [InSPIRE].

[5] ATLAS collaboration, Observation of associated near-side and away-side long-range correlations in $\sqrt{s_{\mathrm{NN}}}=5.02 \mathrm{TeV}$ proton-lead collisions with the ATLAS detector, Phys. Rev. Lett. 110 (2013) 182302 [arXiv:1212.5198] [INSPIRE].

[6] CMS collaboration, Observation of long-range near-side angular correlations in proton-lead collisions at the LHC, Phys. Lett. B 718 (2013) 795 [arXiv:1210.5482] [INSPIRE].

[7] ALICE collaboration, Long-range angular correlations on the near and away side in $\mathrm{p}-\mathrm{Pb}$ collisions at $\sqrt{s_{\mathrm{NN}}}=5.02 \mathrm{TeV}$, Phys. Lett. B 719 (2013) 29 [arXiv:1212.2001] [INSPIRE].

[8] CMS collaboration, Evidence for collectivity in pp collisions at the LHC, Phys. Lett. B $\mathbf{7 6 5}$ (2017) 193 [arXiv:1606.06198] [INSPIRE].

[9] M.P. Heller, R. Jefferson, M. Spaliński and V. Svensson, Hydrodynamic attractors in phase space, arXiv: 2003.07368 [INSPIRE].

[10] M.P. Heller and M. Spalinski, Hydrodynamics beyond the gradient expansion: resurgence and resummation, Phys. Rev. Lett. 115 (2015) 072501 [arXiv:1503.07514] [INSPIRE]. 
[11] M.P. Heller and V. Svensson, How does relativistic kinetic theory remember about initial conditions?, Phys. Rev. D 98 (2018) 054016 [arXiv:1802.08225] [InSPIRE].

[12] M.P. Heller, A. Kurkela, M. Spaliński and V. Svensson, Hydrodynamization in kinetic theory: transient modes and the gradient expansion, Phys. Rev. D 97 (2018) 091503 [arXiv: 1609.04803] [INSPIRE].

[13] M.P. Heller, R.A. Janik and P. Witaszczyk, Hydrodynamic gradient expansion in gauge theory plasmas, Phys. Rev. Lett. 110 (2013) 211602 [arXiv: 1302.0697] [INSPIRE].

[14] M. Shokri and F. Taghinavaz, Conformal Bjorken flow in the general frame and its attractor: similarities and discrepancies with the Müller-Israel-Stewart formalism, arXiv:2002.04719 [INSPIRE].

[15] M. McNelis and U. Heinz, Hydrodynamic generators in relativistic kinetic theory, Phys. Rev. C 101 (2020) 054901 [arXiv:2001.09125] [InSPIRE].

[16] G.S. Denicol and J. Noronha, Hydrodynamic attractor and the fate of perturbative expansions in Gubser flow, Phys. Rev. D 99 (2019) 116004 [arXiv: 1804.04771] [InSPIRE].

[17] J.-P. Blaizot and L. Yan, Fluid dynamics of out of equilibrium boost invariant plasmas, Phys. Lett. B $\mathbf{7 8 0}$ (2018) 283 [arXiv:1712.03856] [INSPIRE].

[18] M. Strickland, J. Noronha and G. Denicol, Anisotropic nonequilibrium hydrodynamic attractor, Phys. Rev. D 97 (2018) 036020 [arXiv:1709.06644] [InSPIRE].

[19] I. Aniceto and M. Spaliński, Resurgence in extended hydrodynamics, Phys. Rev. D 93 (2016) 085008 [arXiv:1511.06358] [InSPIRE].

[20] G. Basar and G.V. Dunne, Hydrodynamics, resurgence and transasymptotics, Phys. Rev. D 92 (2015) 125011 [arXiv: 1509.05046] [InSPIRE].

[21] W.A. Hiscock and L. Lindblom, Generic instabilities in first-order dissipative relativistic fluid theories, Phys. Rev. D 31 (1985) 725 [INSPIRE].

[22] W.A. Hiscock and L. Lindblom, Linear plane waves in dissipative relativistic fluids, Phys. Rev. D 35 (1987) 3723 [inSPIRE].

[23] W. Israel, Nonstationary irreversible thermodynamics: a causal relativistic theory, Annals Phys. 100 (1976) 310 [INSPIRE].

[24] W.A. Hiscock and L. Lindblom, Stability and causality in dissipative relativistic fluids, Annals Phys. 151 (1983) 466 [INSPIRE].

[25] S. Pu, T. Koide and D.H. Rischke, Does stability of relativistic dissipative fluid dynamics imply causality?, Phys. Rev. D 81 (2010) 114039 [arXiv:0907.3906] [InSPIRE].

[26] G.S. Denicol, T. Kodama, T. Koide and P. Mota, Stability and causality in relativistic dissipative hydrodynamics, J. Phys. G 35 (2008) 115102 [arXiv:0807.3120] [InSPIRE].

[27] S. Floerchinger and E. Grossi, Causality of fluid dynamics for high-energy nuclear collisions, JHEP 08 (2018) 186 [arXiv:1711.06687] [INSPIRE].

[28] P. Kovtun, First-order relativistic hydrodynamics is stable, JHEP 10 (2019) 034 [arXiv: 1907.08191] [INSPIRE].

[29] F.S. Bemfica, M.M. Disconzi and J. Noronha, Nonlinear causality of general first-order relativistic viscous hydrodynamics, Phys. Rev. D 100 (2019) 104020 [arXiv:1907.12695] [INSPIRE]. 
[30] F.S. Bemfica, M.M. Disconzi and J. Noronha, Causality and existence of solutions of relativistic viscous fluid dynamics with gravity, Phys. Rev. D 98 (2018) 104064 [arXiv: 1708.06255] [INSPIRE].

[31] K. Jensen, R. Loganayagam and A. Yarom, Thermodynamics, gravitational anomalies and cones, JHEP 02 (2013) 088 [arXiv:1207.5824] [INSPIRE].

[32] U. Moitra, S.K. Sake and S.P. Trivedi, Near-extremal fluid mechanics, arXiv:2005.00016 [INSPIRE].

[33] K. Jensen, M. Kaminski, P. Kovtun, R. Meyer, A. Ritz and A. Yarom, Towards hydrodynamics without an entropy current, Phys. Rev. Lett. 109 (2012) 101601 [arXiv: 1203.3556] [INSPIRE].

[34] N. Abbasi, K. Naderi and F. Taghinavaz, Hydrodynamic excitations from chiral kinetic theory and the hydrodynamic frames, JHEP 03 (2018) 191 [arXiv:1712.06175] [INSPIRE].

[35] I.S. Gradshteyn and I.M. Ryznik, Table of integrals, series and products, seventh edition, Elsevier/Academic Press, Amsterdam, The Netherlands (2007). 